TRANSACTIONS OF THE

AMERICAN MATHEMATICAL SOCIETY

Volume 357, Number 2, Pages 669-694

S 0002-9947(04)03540-8

Article electronically published on April 16, 2004

\title{
DYNAMICAL SYSTEMS DISJOINT FROM ANY MINIMAL SYSTEM
}

\author{
WEN HUANG AND XIANGDONG YE
}

\begin{abstract}
Furstenberg showed that if two topological systems $(X, T)$ and $(Y, S)$ are disjoint, then one of them, say $(Y, S)$, is minimal. When $(Y, S)$ is nontrivial, we prove that $(X, T)$ must have dense recurrent points, and there are countably many maximal transitive subsystems of $(X, T)$ such that their union is dense and each of them is disjoint from $(Y, S)$. Showing that a weakly mixing system with dense periodic points is in $\mathcal{M}^{\perp}$, the collection of all systems disjoint from any minimal system, Furstenberg asked the question to characterize the systems in $\mathcal{M}^{\perp}$. We show that a weakly mixing system with dense regular minimal points is in $\mathcal{M}^{\perp}$, and each system in $\mathcal{M}^{\perp}$ has dense minimal points and it is weakly mixing if it is transitive. Transitive systems in $\mathcal{M}^{\perp}$ and having no periodic points are constructed. Moreover, we show that there is a distal system in $\mathcal{M}^{\perp}$.

Recently, Weiss showed that a system is weakly disjoint from all weakly mixing systems iff it is topologically ergodic. We construct an example which is weakly disjoint from all topologically ergodic systems and is not weakly mixing.
\end{abstract}

\section{§1. INTRODUCTION}

By a topological dynamical system (TDS for short) $(X, T)$ we mean a compact metric space $X$ with a continuous surjective map $T$ from $X$ to itself. Recall that $(X, T)$ is transitive if for each pair of open (i.e., nonempty and open) subsets $U$ and $V, N(U, V)=\left\{n \in \mathbb{Z}_{+}: T^{-n} V \cap U \neq \emptyset\right\}$ is infinite. $(X, T)$ is weakly mixing if $(X \times X, T \times T)$ is transitive. $x \in X$ is a transitive point if $\left\{T(x), T^{2}(x), \ldots\right\}$ is dense in $X$. It is well known if $(X, T)$ is transitive, then the set of transitive points is a dense $G_{\delta}$ set (denoted by $\operatorname{Tran}_{T}$ ) and if $\operatorname{Tran}_{T}=X$, we say that $(X, T)$ is minimal. For a minimal system $(X, T)$ each point of $X$ is called a minimal point. The orbit of $x, \operatorname{orb}(x, T)$, is the set $\{x, T(x), \ldots\}$. The $\omega$-limit set of $x, \omega(x, T)$, is the set $\bigcap_{n>0} \operatorname{cl}\left(\left\{T^{i}(x): i \geq n\right\}\right)$.

Let $\mathcal{M}$ be the collection of all minimal systems. The notion of disjointness of two TDS was introduced in $\mathrm{F}$. If $(X, T)$ and $(Y, S)$ are two TDS, we say $J \subset X \times Y$ is a joining of $X$ and $Y$ if $J$ is a nonempty closed invariant set and is projected onto $X$ and $Y$. If each joining is equal to $X \times Y$, we then say that $(X, T)$ and $(Y, S)$ are disjoint or $(X, T) \perp(Y, S)$ or $X \perp Y$. Furstenberg [F] showed that if two systems are disjoint, then one of them is minimal. Thus the most natural

Received by the editors November 1, 2002 and, in revised form, July 15, 2003.

2000 Mathematics Subject Classification. Primary 54H20; Secondary 58K15.

Key words and phrases. Disjoint, weakly disjoint, minimal, scattering, weakly mixing.

The research of the second author was supported by the 973 project. 
question is to characterize $\mathcal{M}^{\perp}$ (Question $\mathbf{E}$ in $[\mathbf{F}]$ ). It is known $[\mathrm{F}]$ that a weakly mixing system with dense periodic points is in $\mathcal{M}^{\perp}$. We show that a weakly mixing system with dense regular minimal points is in $\mathcal{M}^{\perp}$, and each system in $\mathcal{M}^{\perp}$ has dense minimal points and it is weakly mixing if it is transitive. Transitive systems in $\mathcal{M}^{\perp}$ and having no periodic points are constructed. Moreover, we show that if $(X, T) \perp(Y, S)$, and $(Y, S)$ is nontrivial minimal, then $(X, T)$ must have a dense set of recurrent points, and there are countably many maximal transitive subsystems of $(X, T)$ such that their union is dense and each of them is disjoint from $(Y, S)$. Furthermore, we show that there is a distal system in $\mathcal{M}^{\perp}$, and if $(X, T)$ is equicontinuous and in $\mathcal{M}^{\perp}$, then $(X, T)$ is consisting of fixed points.

A TDS $(X, T)$ is an E-system if it is transitive and there is an invariant measure $\mu$ with full support, i.e., $\operatorname{supp}(\mu)=X ;(X, T)$ is an $M$-system if it is transitive and the set of minimal points is dense (for some special $M$-systems see [DY]); and $(X, T)$ is topologically ergodic (TE, for short) if $(X, T)$ is transitive and for each nonempty open subsets $U, V$ of $X, N(U, V)$ is syndetic, i.e., with bounded gaps. It is known that a minimal system is an $E$-system, and an $E$-system is TE GH2. Two TDS are weakly disjoint if their product is transitive. Note that if both $(X, T)$ and $(Y, S)$ are transitive and $(X, T) \perp(Y, S)$, then they are weakly disjoint. Call a system

- extremely scattering if it is weakly disjoint from all TE systems,

- strongly scattering if it is weakly disjoint from all $E$-systems,

- scattering if it is weakly disjoint from all minimal systems, and

- weakly scattering if it is weakly disjoint from all minimal equicontinuous systems.

Note that weak mixing implies extreme scattering as for a weakly mixing system $N(U, V)$ is thick, i.e., it contains arbitrary long intervals of natural numbers $[\mathrm{F}$. Recently Weiss [We] showed that a system is weakly disjoint from all weakly mixing systems iff it is TE (for the recent development related to this result see $[\mathrm{SY}]$ ). We construct an example which is extremely scattering and is not weakly mixing. Note that in $[\mathrm{AG}$ the authors showed that weak mixing and scattering are different properties, and in [HY] we have showed that extreme scattering and strong scattering are different properties. It remains open if strong scattering, scattering and weak scattering are different properties.

For a TDS $(X, T)$ with a metric $d$, we say $(\widetilde{X}, \widetilde{T})$ is the natural extension of $(X, T)$, if $\widetilde{X}=\left\{\left(x_{1}, x_{2}, \cdots\right): T\left(x_{i+1}\right)=x_{i}, x_{i} \in X, i \in \mathbb{N}\right\}$, which is a subspace of the product space $\prod_{i=1}^{\infty} X$ with the compatible metric $d_{T}$ defined by

$$
d_{T}\left(\left(x_{1}, x_{2}, \cdots\right),\left(y_{1}, y_{2}, \cdots\right)\right)=\sum_{i=1}^{\infty} \frac{d\left(x_{i}, y_{i}\right)}{2^{i}} .
$$

Moreover, $\widetilde{T}: \widetilde{X} \longrightarrow \widetilde{X}$ is the shift homeomorphism, i.e., $\widetilde{T}\left(x_{1}, x_{2}, \cdots\right)=$ $\left(T\left(x_{1}\right), x_{1}, x_{2}, \cdots\right)$. Let $\pi_{i}: \widetilde{X} \longrightarrow X$ be the projection to the $i$-th coordinate. To end the section we prove first that when considering disjointness we may assume each map is a homeomorphism, and then prove a disjoint theorem for distal systems.

Proposition 1.1. Let $(X, T),(Y, S)$ and $(Z, W)$ be $T D S$.

(1) If $(X, T) \perp(Y, S)$ and $(Z, W)$ is a factor of $(X, T)$, then $(Z, W) \perp(Y, S)$. 
(2) $(X, T) \perp(Y, S)$ iff $(\widetilde{X}, \widetilde{T}) \perp(\widetilde{Y}, \widetilde{S})$, where $(\widetilde{X}, \widetilde{T})$ and $(\widetilde{Y}, \widetilde{S})$ are the natural extensions of $(X, T)$ and $(Y, S)$ respectively.

(3) $\mathcal{M}^{\perp \perp}=\mathcal{M}$.

Proof. (1) Let $\pi:(X, T) \rightarrow(Z, W)$ be the factor map. Let $J$ be a joining of $(Z, W)$ and $(Y, S)$. Then $J^{\prime}=\{(x, y) \in X \times Y:(\pi(x), y) \in J\}$ is a joining of $(X, T)$ and $(Y, S)$. Since $(X, T) \perp(Y, S), J^{\prime}=X \times Y$. Moreover, $J=\pi \times i d\left(J^{\prime}\right)=Z \times Y$, hence $(Z, W) \perp(Y, S)$.

(2) $(\Rightarrow)$ For each $i \in \mathbb{N}$ let $\pi_{i}: \widetilde{X} \longrightarrow X$ and $\phi_{i}: \widetilde{Y} \longrightarrow Y$ be the projections to the $i$-th coordinates respectively. Then $(\widetilde{X} \times \widetilde{Y}, \widetilde{T} \times \widetilde{S})$ is a natural extension of $X \times Y$ and $\pi_{i} \times \phi_{i}$ is the projection to the $i$-th coordinate.

Let $\widetilde{J}$ be a joining of $(\widetilde{X}, \widetilde{T})$ and $(\widetilde{Y}, \widetilde{S})$, and $J_{i}=\pi_{i} \times \phi_{i}(\widetilde{J}), i \in \mathbb{N}$. Then $J_{i}$ is a joining of $(X, T)$ and $(Y, S)$. It follows that $J_{i}=X \times Y$ and consequently $\widetilde{J}=\widetilde{X} \times \widetilde{Y}$. That is, $(\widetilde{X}, \widetilde{T}) \perp(\widetilde{Y}, \widetilde{S})$.

$(\Leftarrow)$ It follows immediately from $(1)$.

(3) It follows just because there exists some nonminimal system in $\mathcal{M}^{\perp}$, e.g., weakly mixing systems with dense periodic points and any system disjoint from a nonminimal system is minimal.

Remark 1.2. In $\mathrm{AG}$, Akin and Glasner introduced the notion of residual property. Disjointness from all minimal systems is a residual property by Theorem 2.6 in [AG].

Let $\mathcal{D}$ be the collection of all distal systems. The following theorem was proved in $[\mathrm{P}]$ and for completeness we include a proof.

Theorem 1.3. A dynamical system is in $\mathcal{D}^{\perp}$ iff it is minimal and weakly mixing.

Proof. Assume that $(X, T) \perp \mathcal{D}$. As there are nonminimal distal systems, $(X, T)$ is minimal. Let $(Y, S)$ be the maximal equicontinuous factor of $(X, T)$. Then $(X, T) \perp(Y, S)$. This shows that $(Y, S) \perp(Y, S)$ and thus $(Y, S)$ is trivial. Hence by [M] $(X, T)$ is weakly mixing.

Now let $(X, T)$ be minimal and weakly mixing, and $(Y, S)$ be distal. It is known that $(Y, S)$ is a union of minimal distal subsets. Let $J \subset X \times Y$ be a joining. Assume $x \in X, y \in Y$ and $M_{y}$ is the orbit closure of $y$. It is easy to see that $J_{y}=\operatorname{orb}((x, y), T \times S) \subset J$ and is a joining of $(X, T)$ and $\left(M_{y}, S\right)$. It is known $\mathrm{F}$. that $(X, T) \perp\left(M_{y}, S\right)$. Thus $J_{y}=X \times M_{y} \subset J$ which implies $J=X \times Y$.

\section{§2. Transitive Systems Disjoint from any minimal System}

In [F] Furstenberg asked the question to characterize systems disjoint from any distal or minimal system. Theorem 1.3 gives a complete answer to the first part of the question. In Sections 2, 3 and 4 we provide a partial answer to the second part. We will give some necessary conditions and some sufficient conditions for membership in $\mathcal{M}^{\perp}$.

If $(X, T)$ is a TDS and $x$ is a minimal point of $T$, then for each neighborhood $U$ of $x, N(x, U)=\left\{n \in \mathbb{Z}_{+}: T^{n}(x) \in U\right\}$ is syndetic [GH2]. Note that a subset $A$ of $\mathbb{Z}_{+}$is piecewise syndetic if it is the intersection of a syndetic set with a thick set, and it is thickly syndetic if for each $n \in \mathbb{N}$ there is a syndetic subset $\left\{w_{1}^{n}, w_{2}^{n}, \ldots\right\}$ of $A$ such that $\left\{w_{i}^{n}, w_{i}^{n}+1, \ldots, w_{i}^{n}+n\right\} \subset A$ for each $i$. For further investigation we need the following lemma. 
Lemma 2.1. Let $(X, T)$ be a transitive system and $x \in \operatorname{Tran}_{T}$. Then

(1) $(X, T)$ is an $M$-system if and only if for each neighborhood $U$ of $x, N(x, U)$ is piecewise syndetic.

(2) Let $K$ be a minimal set of $(X, T)$. Then $(X, T)$ has only one minimal set iff for each neighborhood $U$ of $K, N(x, U)$ is thickly syndetic.

Proof. (1) If $(X, T)$ is an $M$-system, it is clear that for each neighborhood $U$ of $x, N(x, U)$ is piecewise syndetic, since for each minimal point $y \in U, N(y, U)$ is syndetic and there are $\left\{n_{i}\right\}$ such that $T^{n_{i}}(x) \rightarrow y$.

Now we assume that for each neighborhood $U$ of $x, N(x, U)$ is piecewise syndetic. Let $\epsilon>0$ with $\operatorname{cl}\left(B_{\epsilon / 2}(x)\right) \subset U$. Thus there are $p \in \mathbb{N}$ and $\left\{m_{j}^{i}: i \in \mathbb{N}\right.$ and $1 \leq j \leq$ i\} $\subset N\left(x, B_{\epsilon / 2}(x)\right)$ such that $m_{1}^{i}<\ldots<m_{i}^{i}$ and $m_{j+1}^{i}-m_{j}^{i} \leq p$ for $1 \leq j \leq i-1$. Let $y$ be a limit point of $\left\{T^{m_{1}^{i}}(x)\right\}$. Then it is clear that $N(y, U)$ is syndetic. Let $M$ be a minimal set in the orbit closure of $y$ under $T$. We claim that $M \cap \operatorname{cl}(U) \neq \emptyset$. In fact, if $M \cap \operatorname{cl}(U)=\emptyset$, then there is an open set $V \supset M$ with $U \cap V=\emptyset$. It is clear that $N(y, V)$ is thick. Thus $N(y, U)$ is not syndetic, a contradiction.

Since $M \cap \operatorname{cl}(U) \neq \emptyset$, it follows by the transitivity of $(X, T)$ that $(X, T)$ is an $M$-system.

(2) Assume that $(X, T)$ has only one minimal set $K$. For each neighborhood $U$ of $K$, let $U_{i} \subset U$ be a neighborhood of $K$ such that if $T^{j}(x) \in U_{i}$, then $T^{j+k}(x) \in U$ for each $1 \leq k \leq i$. As $N\left(x, U_{i}\right)$ is syndetic for each $i$, we have that $N(x, U)$ is thickly syndetic.

Conversely assume that $T$ has a minimal set $K_{1}$ with $K_{1} \cap K=\emptyset$. Then $N(x, V)$ is thick for each neighborhood $V$ of $K_{1}$ disjoint from $U$. Then $N(x, U) \subset$ $\mathbb{Z}_{+} \backslash N(x, V)$ cannot be syndetic, a contradiction.

Definition. A subset $A$ of $\mathbb{Z}_{+}$is called an $m$-set if there exist a minimal system $(Y, S), y \in Y$, and an open subset $V$ of $Y$ such that $A \supset N(y, V)$.

For a transitive system whether it is in $\mathcal{M}^{\perp}$ can be checked through $m$-sets as the following theorem shows. For a minimal dynamical system $(Y, S)$, we define

$$
\begin{aligned}
& \mathcal{F}_{Y}=\left\{A \subset \mathbb{Z}_{+}: A \supset N(y, V) \text { for some } y \in Y \text { and open subset } V\right\} \text { and } \\
& k \mathcal{F}_{Y}=\left\{B \subset \mathbb{Z}_{+}: B \cap A \neq \emptyset \text { for each } A \in \mathcal{F}_{Y}\right\} .
\end{aligned}
$$

Theorem 2.2. Let $(X, T)$ be a transitive system and $x \in$ Trans $_{T}$. Then

(1) $(X, T) \in \mathcal{M}^{\perp}$ iff $N(x, U) \cap A \neq \emptyset$ for any neighborhood $U$ of $x$ and any $m$-set $A$.

(2) $(X, T) \perp(Y, S)$ iff for any open neighborhood $U$ of $x$, one has $N(x, U) \in$ $k \mathcal{F}_{Y}$.

Proof. We show (1), and (2) is similar.

Let $(X, T) \in \mathcal{M}^{\perp}$. For any $m$-set $A$, there exist a minimal system $(Y, S), y \in Y$, and an open subset $V$ of $Y$ such that $A \supset N(y, V)$.

Set $J=\operatorname{cl}(\operatorname{orb}((x, y), T \times S))$. Then $J$ is a joining of $(X, T)$ and $(Y, S)$. Since $(X, T) \perp(Y, S), J=X \times Y$. Hence for any neighborhood $U$ of $x$ one has $N((x, y), U \times V) \neq \emptyset$, i.e., $N(x, U) \cap A \neq \emptyset$.

Conversely, let $(Y, S)$ be a minimal system and $J$ a joining of $(X, T)$ and $(Y, S)$. It is clear that, there is $y \in Y$ with $(x, y) \in J$. For any neighborhood $U$ of $x$ and any open subset $V$ of $Y$, one has $N(x, U) \cap N(y, V) \neq \emptyset$. This implies 
$\operatorname{cl}(\operatorname{orb}((x, y), T \times S)) \cap(U \times V) \neq \emptyset$. Since $\operatorname{cl}(\operatorname{orb}((x, y), T \times S))$ is $T \times S$-invariant and closed, one has $\operatorname{cl}(\operatorname{orb}((x, y), T \times S))=X \times Y$. Thus $J=X \times Y$.

For any $S \subset \mathbb{Z}_{+}$let $1_{S}$ be the indication function from $\mathbb{Z}_{+}$to $\{0,1\}$, i.e., $1_{S}(s)=1$ if $s \in S$ and $1_{S}(s)=0$ if $s \notin S$. Note that if $s=(s(0), s(1), \ldots) \in \Sigma=\{0,1\}^{\mathbb{Z}_{+}}$, then we use $s[n ; m]$ to denote $(s(n), s(n+1), \ldots, s(m))$ whenever $n \leq m$. Let $\sigma: \Sigma \longrightarrow \Sigma$ be the shift map and, for a finite word $A$, let $|A|$ stand for the length of $A$.

Let $\mathcal{P}$ be the collection of all subsets of $\mathbb{Z}_{+}$. A subset $\mathcal{F}$ of $\mathcal{P}$ is a family, if it is hereditary upwards. That is, $F_{1} \subset F_{2}$ and $F_{1} \in \mathcal{F}$ imply $F_{2} \in \mathcal{F}$. Any subset $\mathcal{A}$ of $\mathcal{P}$ can generate a family $[\mathcal{A}]=\{F \in \mathcal{P}: F \supset A$ for some $A \in \mathcal{A}\}$.

Clearly, all $m$-sets form a family, we call it the family of $m$-sets. Now, we have

Proposition 2.3. The family of $m$-sets is the family generated by the sets whose indicator functions are the minimal points of $(\Sigma, \sigma)$.

Proof. We denote the family generated by the sets whose indicator functions are the minimal points of $(\Sigma, \sigma)$ by $\mathcal{F}_{m}$. Clearly, if $1_{F}$ is the indicator function of $F$, then $F=N\left(1_{F},[1]\right)$, where [1] $=\{s \in \Sigma: s(0)=1\}$. Hence $\mathcal{F}_{m}$ is contained in the family of $m$-sets.

On the other hand, let $A$ be an $m$-set. Then there exist a minimal system $(X, T)$ with metric $d, x \in X$ and an open subset $V$ of $X$ such that $A \supset N(x, V)$. It is easy to see that we can shrink $V$ to an open subset $V^{\prime}$ whose boundary is disjoint from the orbit of $x$.

Then do the classical lifting trick, a la Glasner, Adler, etc. Let

$$
\begin{aligned}
Y=\left\{(z, t) \in X \times \Sigma: t(i)=1 \text { implies } T^{i} z \in \operatorname{cl}\left(V^{\prime}\right)\right. \\
\\
\text { and } \left.t(i)=0 \text { implies } T^{i} z \in \operatorname{cl}\left(X \backslash V^{\prime}\right)\right\} .
\end{aligned}
$$

Then $Y$ is a $T \times \sigma$-invariant closed subset of $X \times \Sigma$. Since the orbit of $x$ doesn't meet the boundary of $V^{\prime}$, there is a unique $t \in \Sigma$ such that $(x, t) \in Y$ and $t$ is the indicator function of $N\left(x, V^{\prime}\right)$. Take a minimal subset $J$ of $(Y, T \times \sigma)$ with $J \subset \operatorname{cl}(\operatorname{orb}((x, t), T \times \sigma))$ and let $\pi_{X}: J \rightarrow X$ be the projective map. Since $(X, T)$ is minimal, $\pi_{X}(J)=X$. Hence $(x, t) \in J$. Projecting $J$ to $\Sigma$ we see that $t$ is a minimal point. Hence $A \in \mathcal{F}_{m}$ as $A \supset N\left(x, V^{\prime}\right)$ and $t=1_{N\left(x, V^{\prime}\right)}$.

The following theorem is crucial for this section.

Theorem 2.4. Every thickly syndetic set contains an $m$-set.

Proof. Let $F \subset \mathbb{Z}_{+}$be a thickly syndetic subset. We will construct $y^{n}=1_{F_{n}} \in$ $\{0,1\}^{\mathbb{Z}_{+}}$such that $F_{n} \subset F$ and $y=\lim y^{n}=1_{A}$ is a minimal point. Let $Y=$ $\operatorname{cl}(\operatorname{orb}(y, \sigma))$ and $[1]=\{x \in Y: x(0)=1\}$. As $A \subset F$ and $A=N(y,[1])$, the theorem follows.

To obtain $y^{n}$ we construct a finite word $A_{n}$ such that $y^{n}$ begins with $A_{n}, A_{n}$ appears in $y^{n}$ syndetically and $A_{n+1}$ begins with $A_{n}$. The reason we can do this is that $1^{n}=(1, \ldots, 1)$ ( $n$ times) appears in $1_{F}$ syndetically for each $n \in \mathbb{N}$. More precisely we do as follows.

Step 1. Construct $A_{1}$ and $F_{1} \subset F$ such that $A_{1}$ appears in $y^{1}=1_{F_{1}}$ with gaps bounded by $l_{1}$ and $y^{1}$ begins with $A_{1}$.

Let $\min F=k_{1}-1$ and $A_{1}=1_{F}\left[0 ; k_{1}-1\right]$. Set $r_{1}=k_{1}$. As $F$ is thickly syndetic, $1^{r_{1}}$ appears in $F$ at a syndetic set $W_{1}=\left\{w_{1}^{1}, w_{2}^{1}, \ldots\right\}$. Without loss of generality, 
assume that $2 r_{1} \leq w_{j+1}^{1}-w_{j}^{1} \leq l_{1}$ and $2 k_{1} \leq w_{1}^{1} \leq l_{1}$, where $l_{1}$ is some number in $\mathbb{N}$. Put $u_{i}^{1}=w_{i}^{1}, i \in \mathbb{N}$. Choose $y^{1} \in\{0,1\}^{\mathbb{Z}_{+}}$such that

$$
y^{1}\left[0 ; k_{1}-1\right]=A_{1}, \quad y^{1}\left[u_{i}^{1} ; u_{i}^{1}+k_{1}-1\right]=A_{1}
$$

and

$$
y^{1}(j)=0 \quad \text { if } j \in \mathbb{Z}_{+} \backslash\left(\left[0 ; k_{1}-1\right] \cup \bigcup_{i=1}^{\infty}\left[u_{i}^{1} ; u_{i}^{1}+k_{1}-1\right]\right) .
$$

It is easy to see that $A_{1}$ appears in $y^{1}$ with gaps bounded by $l_{1}$ and $F_{1} \subset F$, where $1_{F_{1}}=y^{1}$.

Step 2. Construct $A_{2}$ and $F_{2} \subset F$ such that

(1) $A_{2}$ has the form of $A_{1} V_{1} A_{1}$ and if $k_{2}=\left|A_{2}\right|$, then $A_{2}=y^{1}\left[0 ; k_{2}-1\right]$.

(2) $y^{2}\left[0 ; k_{2}-1\right]=A_{2}$ and $A_{1}, A_{2}$ appear in $y^{2}$ syndetically with gaps bounded by $l_{1}$ and $l_{2}$ respectively.

(3) $F_{2}=\left\{i \in \mathbb{Z}_{+}: y^{2}(i)=1\right\} \subset F$.

Set $k_{2}=u_{1}^{1}+k_{1}$ and let $A_{2}=y^{1}\left[0 ; k_{2}-1\right]$. Then $A_{2}$ has the form of $A_{1} V_{1} A_{1}$. Let $r_{2}=2 l_{1}+2 k_{1}+k_{2}$. As $F$ is thickly syndetic, $1^{r_{2}}$ appears in $F$ at a syndetic set $W_{2}=\left\{w_{1}^{2}, w_{2}^{2}, \ldots\right\}$. Without loss of generality, assume that $2 r_{2} \leq w_{j+1}^{2}-w_{j}^{2} \leq$ $l_{2}-\left(l_{1}+k_{1}\right)$ and $2 k_{2} \leq w_{1}^{2} \leq l_{2}-\left(l_{1}+k_{1}\right)$, where $l_{2}$ is some number in $\mathbb{N}$.

To get $y^{2}$ we change $y^{1}$ at places $\left[w_{i}^{2} ; w_{i}^{2}+r_{2}-1\right]$ for each $i \in \mathbb{N}$. It is enough to show how this is done at $\left[w_{1}^{2} ; w_{1}^{2}+r_{2}-1\right]$.

Let $k, j$ satisfy that $u_{k-1}^{1}<w_{1}^{2} \leq u_{k}^{1}$ and $u_{j}^{1}+k_{1}-1 \leq w_{1}^{2}+r_{2}-1<u_{j+1}^{1}+k_{1}-1$. Let $l$ be the integer part of $\left(u_{j}^{1}-1-u_{k}^{1}-k_{1}-k_{2}\right) / k_{1}$.

Put $u_{1}^{2}=u_{k}^{1}+k_{1}$. Let $y^{2}\left[u_{1}^{2} ; u_{1}^{2}+k_{2}-1\right]=A_{2}$ and $y^{2}\left[u_{1}^{2}+k_{2}+p k_{1} ; u_{1}^{2}+k_{2}+\right.$ $\left.(p+1) k_{1}-1\right]=A_{1}$ for $p=0,1, \ldots, l-1$. That is, first we put $A_{2}$ at place $u_{1}^{2}$ and then we put as many $A_{1}$ as we can. We do the same at all places $\left[w_{i}^{2} ; w_{i}^{2}+r_{2}-1\right]$; we get $u_{i}^{2} \in\left[w_{i}^{2}, w_{i}^{2}+r_{2}-1\right]$ with $y^{2}\left[u_{i}^{2} ; u_{i}^{2}+k_{2}-1\right]=A_{2}, i=1,2, \ldots$

In such a way we get $y^{2}$. It is easy to see that $y^{1}$ and $y^{2}$ differ possibly at $\left[w_{i}^{2} ; w_{i}^{2}+r_{2}-1\right]$. Thus

$$
F_{2}=\left\{i \in \mathbb{Z}_{+}: y^{2}(i)=1\right\} \subset F_{1} \cup \bigcup_{i=1}^{\infty}\left[w_{i}^{2} ; w_{i}^{2}+r_{2}-1\right] .
$$

At the same time $A_{1}, A_{2}$ appear in $y^{2}$ syndetically with gaps bounded by $l_{1}$ and $l_{2}$ respectively by the construction.

Step 3. Construct $A_{m+1}$ and $F_{m+1} \subset F$ inductively such that

(1) $A_{m+1}$ has the form of $A_{m} V_{m} A_{m}$ and if $k_{m+1}=\left|A_{m+1}\right|$, then $A_{m+1}=$ $y^{m}\left[0 ; k_{m+1}-1\right]$.

(2) $y^{m+1}\left[0 ; k_{m+1}-1\right]=A_{m+1}$ and $A_{i}$ appear in $y^{m+1}$ syndetically with gaps bounded by $l_{i}$ for each $1 \leq i \leq m+1$.

(3) $F_{m+1}=\left\{i \in \mathbb{Z}_{+}: y^{m+1}(i)=1\right\} \subset F$.

Set $k_{m+1}=u_{1}^{m}+k_{m}$ and let $A_{m+1}=y^{m}\left[0 ; k_{m+1}-1\right]$. Then $A_{m+1}$ has the form of $A_{m} V_{m} A_{m}$. Let $r_{m+1}=2 l_{m}+2 k_{m}+k_{m+1}$. As $F$ is thickly syndetic, $1^{r_{m+1}}$ appears in $F$ at a syndetic set $W_{m+1}=\left\{w_{1}^{m+1}, w_{2}^{m+1}, \ldots\right\}$. Without loss of generality, assume that $2 r_{m+1} \leq w_{j+1}^{m+1}-w_{j}^{m+1} \leq l_{m+1}-\left(l_{m}+k_{m}\right)$ and $2 k_{m+1} \leq$ $w_{1}^{m+1} \leq l_{m+1}-\left(l_{m}+k_{m}\right)$, where $l_{m+1}$ is some number in $\mathbb{N}$.

To get $y^{m+1}$ we change $y^{m}$ at places $\left[w_{i}^{m+1} ; w_{i}^{m+1}+r_{m+1}-1\right]$ for each $i \in \mathbb{N}$. It is enough to show how this is done at $\left[w_{1}^{m+1} ; w_{1}^{m+1}+r_{m+1}-1\right]$. 
Let $k, j$ satisfy that $u_{k-1}^{m}<w_{1}^{m+1} \leq u_{k}^{m}$ and $u_{j}^{m}+k_{m}-1 \leq w_{1}^{m+1}+r_{m+1}-1<$ $u_{j+1}^{m}+k_{m}-1$.

Put $u_{1}^{m+1}=u_{k}^{m}+k_{m}$. Let $y^{m+1}\left[u_{1}^{m+1} ; u_{1}^{m+1}+k_{m+1}-1\right]=A_{m+1}$ and

$$
y^{m+1}\left[u_{1}^{m+1}, u_{j}^{m}-1\right]=A_{m+1}\left(A_{m}\right)^{p_{m}}\left(A_{m-1}\right)^{p_{m-1}} \ldots\left(A_{1}\right)^{p_{1}} B_{m+1},
$$

where $B_{m+1}$ is a word and $p_{1}, \ldots, p_{m}$ are natural numbers with

$\left|B_{m+1}\right|<k_{1}$,

$\left|B_{m+1}\right|+k_{1} p_{1}<k_{2}$, and

$\left|B_{m+1}\right|+k_{1} p_{1}+\ldots+k_{i} p_{i}<k_{i+1}$ for each $1 \leq i \leq m-1$.

That is, first we put $A_{m+1}$ at place $u_{1}^{m+1}$ and start from $u_{1}^{m+1}+k_{m+1}$ to $u_{j}^{m}$; we put as many $A_{m}$ as we can and then we put as many $A_{m-1}$ as we can and so on. We do the same at all places $\left[w_{i}^{m+1} ; w_{i}^{m+1}+r_{m+1}-1\right]$; we get $u_{i}^{m+1} \in$ $\left[w_{i}^{m+1} ; w_{i}^{m+1}+r_{m+1}-1\right]$ with $y^{m+1}\left[u_{i}^{m+1} ; u_{i}^{m+1}+k_{m+1}-1\right]=A_{m+1}, i=1,2, \ldots$.

In such a way we get $y^{m+1}$. It is easy to see that $y^{m+1}$ and $y^{m}$ differ possibly only at $\left[w_{i}^{m+1} ; w_{i}^{m+1}+r_{m+1}-1\right], i=1,2, \ldots$. Thus

$$
F_{m+1}=\left\{i \in \mathbb{Z}_{+}: y^{m+1}(i)=1\right\} \subset F_{m} \cup \bigcup_{i=1}^{\infty}\left[w_{i}^{m+1} ; w_{i}^{m+1}+r_{m+1}-1\right] .
$$

At the same time $A_{i}$ appears in $y^{m+1}$ syndetically with gaps bounded by $l_{i}$ for each $1 \leq i \leq m+1$ by the construction.

In such a way for each $m \in \mathbb{N}$ we defined a finite word $A_{m}$. Let $y=\lim A_{m}=$ $\lim y^{m}$. By the construction, $A_{m}$ appears in $y$ with gaps bounded by $l_{m}$ for each $m \in \mathbb{N}$. That is, $y$ is a minimal point for the shift. It is obvious that $y \neq(0,0, \ldots)$. Let $Y=\operatorname{orb}(y, \sigma)$ and $U=\{x \in Y: y(0)=1\}$. Then

$$
\emptyset \neq N(y, U)=\bigcup_{i=1}^{\infty}\left\{i \in \mathbb{Z}_{+}: A_{n}(i)=1,0 \leq i \leq k_{n}-1\right\} \subset \bigcup_{i=1}^{\infty} F_{n} \subset F .
$$

Thus $F$ contains the $m$-set $N(y, U)$.

Remark 2.5. There exists an $m$-set which is not thick. For example, let $(X, T)$ be a nontrivial minimal system and $x \in X$. If $U$ and $V$ are disjoint open subsets of $X$, then $N(x, U)$ and $N(x, V)$ are disjoint $m$-sets. In particular, both are syndetic and so neither is thick.

Now we are ready to show the main result of the section.

Theorem 2.6. Let $(X, T)$ be a transitive TDS. If $(X, T) \perp \mathcal{M}$, then $(X, T)$ is a weakly mixing $M$-system without nontrivial minimal factor.

Proof. Let $x \in \operatorname{Tran}_{T}$ and let $U$ be a nonempty neighborhood $x$. By Theorem 2.2, $N(x, U) \cap A \neq \emptyset$ for any $m$-set $A$. This implies that $N(x, U)$ has nonempty intersection with any thickly syndetic set by Theorem 2.4. Thus $N(x, U)$ is piecewise syndetic. By Lemma $2.1(X, T)$ is an $M$-system. Since a nontrivial system is never disjoint from itself, it follows from Proposition 1.1(1) that a system in $\mathcal{M}^{\perp}$ has only trivial minimal factors. Hence $(X, T)$ has no nontrivial minimal factor.

Since $(X, T)$ is transitive, $(X, T)$ is weakly disjoint from all minimal systems, i.e., $(X, T)$ is scattering $[\mathrm{BHM}]$. By [AG, Theorem $2.9(\mathrm{~b})],(X, T)$ is weakly disjoint from all $M$-systems. Particularly, $(X, T)$ is weakly mixing. 
Remark 2.7. The condition in Theorem 2.6 is not sufficient. For example, let $(X, T)$ be a weakly mixing $M$-system with a fixed point $p$ and let $(Y, S)$ be a minimal strongly mixing system. Then $X \times Y$ is a weakly mixing $M$-system. It is clear that $X \times Y$ is not disjoint from $Y$. Collapsing $\{p\} \times Y$ to a point $p^{\prime}$ we get a system $Z$ which is not disjoint from $Y$. Moreover, $Z$ is a weakly mixing $M$-system without nontrivial minimal factor, as $p^{\prime}$ is a fixed point.

\section{§3. A transitive System in $\mathcal{M}^{\perp}$ without PeRiodic points}

For a transitive system we have obtained some necessary conditions for a system in $\mathcal{M}^{\perp}$. Since Fursternberg has shown that each weakly mixing system with a dense set of periodic points is in $\mathcal{M}^{\perp}$, it is natural to ask if this is a necessary condition. We will give a negative answer by constructing a transitive system in $\mathcal{M}^{\perp}$ without periodic points. In the process to do this, we also obtain some sufficient condition. To start, we first show

Lemma 3.1. Let $A \subset \mathbb{Z}_{+}$be an $m$-set. Then there exists $r \in \mathbb{Z}_{+}$such that $N_{k}(A, r)=\left\{i \in \mathbb{Z}_{+}: k i+r \in A\right\}$ is an m-set and so is syndetic for any $k \in \mathbb{Z}_{+}$.

Proof. Since $A$ is an $m$-set, there exist a minimal system $(X, T), x \in X$, and an open subset $U$ of $X$ such that $A \supset N(x, U)$. Take $r \in \mathbb{Z}_{+}$and a neighborhood $V$ of $x$ such that $T^{r} V \subset U$. Then $N(x, U) \supset N(x, V)+r$. Note that $x$ is also a minimal point of $T^{k}$ for any $k \in \mathbb{Z}_{+}$and thus $\left\{i \in \mathbb{Z}_{+}: T^{k i} x \in V\right\}$ is an $m$-set and so is syndetic. Therefore, $N_{k}(A, r) \supset\left\{i \in \mathbb{Z}_{+}: T^{k i} x \in V\right\}$ is an $m$-set and so is syndetic for any $k \in \mathbb{Z}_{+}$.

Now we give a natural generalization of dense periodic points.

Definition. Let $(X, T)$ be a TDS. We say $(X, T)$ has dense small periodic sets, if for any open subset $U$ of $X$ there exist a nonempty closed $A \subset U$ and $k \in \mathbb{N}$ such that $A$ is invariant for $T^{k}$.

Concerning this notion we have

Proposition 3.2. Let $(X, T)$ be a TDS. Then $(X, T)$ has dense small periodic sets if and only if for any open subset $U$ of $X$ there exist $p \in X, k \in \mathbb{N}$ such that $N_{k}(p, U)=\left\{i \in \mathbb{Z}_{+}: T^{k i} p \in U\right\}$ is thick.

Proof. Let $(X, T)$ have dense small periodic sets. Then for any open subset $U$ of $X$ there exist a closed $A \subset U$ and a positive integer $k$ such that $A$ is invariant for $T^{k}$. Take any $p \in A$; then $N_{k}(p, U)=\mathbb{Z}_{+}$is thick.

Conversely, assume for any open subset $U$ of $X$ there exist $p \in X, k \in \mathbb{N}$ such that $N_{k}(p, U)$ is thick. Let $W$ be an open subset of $X$ and $V$ an open subset with $\operatorname{cl}(V) \subset W$. Then there exist $p \in X$ and $k \in \mathbb{N}$ such that $N_{k}(p, V)$ is thick. Hence we can find $n_{1}<n_{2}<\ldots$ and $y \in X$ such that $T^{k n_{l}} p, T^{k\left(n_{l}+1\right)} p, \cdots, T^{k\left(n_{l}+l\right)} p \in V$ for any $l \in \mathbb{N}$ and $\lim _{l \rightarrow \infty} T^{k n_{l}} p=y$. Clearly, $\operatorname{orb}\left(y, T^{k}\right) \subset \operatorname{cl}(V)$. Let $A=$ $\operatorname{cl}\left(\operatorname{orb}\left(y, T^{k}\right)\right)$. Then $A \subset \operatorname{cl}(V) \subset W$ and $A$ is closed and invariant for $T^{k}$. This shows that $(X, T)$ has dense small periodic sets.

A totally transitive system with dense small periodic sets can be characterized as follows.

Proposition 3.3. Let $(X, T)$ be a TDS. Then $(X, T)$ is a totally transitive system having dense small periodic sets if and only if there exists a transitive point $x$ such 
that for any neighborhood $U$ of $x$ we have property $(\star)$, i.e.,

(*) for any $r \in \mathbb{Z}_{+}$there exists $k \in \mathbb{N}$ such that $N_{k}(x, U, r)=\left\{i \in \mathbb{Z}_{+}\right.$: $\left.T^{k i+r} x \in U\right\}$ is thick.

When these conditions hold then for every $x \in \operatorname{Tran}_{T}$ and every neighborhood $U$ of $x$, condition $(\star)$ holds.

Proof. Let $(X, T)$ be a totally transitive system having dense small periodic sets and $x \in \operatorname{Tran}_{T}$. Then $x \in \operatorname{Tran}_{T^{k}}$ for all $k \in \mathbb{N}$; see [B].

For any neighborhood $U$ of $x$, by Proposition 3.2, there exist $p \in X, k \in \mathbb{N}$ and $n_{1}<n_{2}<\ldots$ such that $N(p, U) \supset \bigcup_{i=1}^{\infty}\left\{k n_{i}, k\left(n_{i}+1\right), \ldots, k\left(n_{i}+i\right)\right\}$. Thus for any natural number $l$ we can find a neighborhood $V_{l}$ of $p$ such that when $y \in V_{l}$, one has $T^{k n_{l}} y, T^{k\left(n_{l}+1\right)} y, \cdots, T^{k\left(n_{l}+l\right)} y \in U$. For any $r \in \mathbb{Z}_{+}$, since $T^{r} x$ is a transitive point of $T^{k}$, there exists $m \in \mathbb{Z}_{+}$with $T^{m k} T^{r} x \in V_{l}$. Moreover, $N_{k}(x, U, r) \supset\left\{n_{l}+m+1, n_{l}+m+2, \cdots, n_{l}+m+l\right\}$ for each $l \in \mathbb{N}$. This shows $N_{k}(x, U, r)$ is thick.

Conversely, assume there exists a transitive point $x$ such that for any neighborhood $U$ of $x$ we have property $(\star)$. To show that $(X, T)$ is a totally transitive system having dense small periodic sets, we need only to show that for any open subset $W$ of $X$ and $n \in \mathbb{N}, N_{n}(x, W) \neq \emptyset$ and there exist $k \in \mathbb{N}$ and $p \in X$ such that $N_{k}(p, W)$ is thick.

Now let $W$ be a given open subset of $X$ and $n \in \mathbb{N}$. Since $x$ is a transitive point, there exists $s \in \mathbb{N}$ such that $T^{s} x \in W$. Take a small open neighborhood $U$ of $x$ with $T^{s} U \subset W$ and $r \in \mathbb{N}$ with $n \mid(r+s)$. For $U$ and $r$, since we have property $(\star)$, there exists $k \in \mathbb{N}$ such that $N_{k}(x, U, r)$ is thick. Let $p=T^{r+s} x$, then $N_{k}(p, W) \supset N_{k}(x, W, r+s) \supset N_{k}(x, U, r)$ as $T^{s} U \subset W$. Therefore, $N_{k}(p, W)$ is thick.

Clearly, $N_{k}(p, W) \cap n \mathbb{N} \neq \emptyset$. For $m \in N_{k}(p, W) \cap n \mathbb{N}$, we have $T^{k m+r+s} x \in W$. As $n \mid(k m+r+s)$, one has $N_{n}(x, W) \neq \emptyset$.

Theorem 3.4. Let $(X, T)$ be a transitive TDS with a transitive point $x$. If for any neighborhood $U$ of $x$ we have property $(\star)$, then $(X, T)$ is in $\mathcal{M}^{\perp}$. That is, if $(X, T)$ is a totally transitive TDS having dense small periodic sets, then $(X, T) \in \mathcal{M}^{\perp}$.

Proof. By Theorem 2.2, it remains to show that $N(x, U) \cap A \neq \emptyset$ for any neighborhood $U$ of $x$ and any $m$-set $A$. By Lemma 3.1, there exists $r \in \mathbb{Z}_{+}$such that $N_{k}(A, r)=\left\{i \in \mathbb{Z}_{+}: k i+r \in A\right\}$ is syndetic for any $k \in \mathbb{Z}_{+}$. Since property ( $\star$ ) holds, there exists $k_{r} \in \mathbb{N}$ such that $N_{k_{r}}(x, U, r)=\left\{i \in \mathbb{Z}_{+}: T^{k_{r} i+r} x \in U\right\}$ is thick. Thus $N_{k_{r}}(x, U, r) \cap N_{k_{r}}(A, r) \neq \emptyset$. This implies that $N(x, U) \cap A \neq \emptyset$.

A minimal point $x$ is regular [GH2] if for each neighborhood $V$ of $x$ there is $k$ such that $N(x, V) \supset k \mathbb{Z}_{+}$.

Clearly, a periodic point is a regular minimal point and a regular minimal point is a minimal point. In order to characterize the minimal system containing a regular minimal point, we need the notions of an almost one-to-one extension and an adding machine.

Let $\pi:(X, T) \rightarrow(Y, S)$ be a factor map. We say $\pi$ is an almost one-to-one extension, if $\operatorname{Inj}_{\pi}=\left\{x \in X: \pi^{-1} \pi(x)=\{x\}\right\}$ is dense in $X$.

Given $K=\left(k_{1}, k_{2}, \cdots\right)$ with $k_{i} \geq 1$, we define $\Sigma_{K}=\prod_{i=1}^{\infty}\left\{0,1, \cdots, k_{i}-1\right\}$, where $\left\{0,1, \cdots, k_{i}-1\right\}$ and $\Sigma_{K}$ are equipped with the discrete and the product topology respectively. If $x=\left(x_{1}, x_{2}, \cdots\right)$ and $y=\left(y_{1}, y_{2}, \cdots\right)$ are two elements 
of $\Sigma_{K}$, then their sum $x \oplus y=\left(z_{1}, z_{2}, \cdots\right)$ is defined as follow. If $x_{1}+y_{1}<k_{1}$, then $z_{1}=x_{1}+y_{1}$; if $x_{1}+y_{1} \geq k_{1}$, then $z_{1}=x_{1}+y_{1}-k_{1}$ and we carry 1 to the next position. The other terms $z_{2}, \cdots$ are successively determined in the same fashion. Let $T_{K}: \Sigma_{K} \rightarrow \Sigma_{K}$ be defined by $T_{K}(z)=z \oplus 1$ for each $z \in \Sigma_{K}$, where $1=(1,0,0, \cdots)$. It is known that $T_{K}$ is a minimal map, which is called an adding machine. We note that if $\left\{i \in \mathbb{N}: k_{i}>1\right\}$ is finite, then $T_{K}$ is periodic and $\Sigma_{K}$ is the unique periodic orbit of $T_{K}$. Now we have

Proposition 3.5. Let $(X, T)$ be a minimal system. Then $(X, T)$ contains a regular minimal point if and only if it is an almost one-to-one extension of an adding machine.

Proof. See the Appendix.

The following is an immediate corollary of Theorem 3.4.

Corollary 3.6. If $(X, T)$ is a totally transitive TDS and has dense periodic points or dense regular minimal points, then $(X, T) \in \mathcal{M}^{\perp}$. Particularly, $(X, T)$ is weakly mixing.

We remark that a nontrivial adding machine or finite system is never totally transitive, and hence the systems described in Corollary 3.6 are never minimal. Moreover, it is known that a totally transitive system having dense periodic points is weakly mixing $[\mathrm{B}]$.

Using Theorem 3.4 we will construct a transitive TDS which is in $\mathcal{M}^{\perp}$ and has no periodic point. Namely, we have

Example 3.7. There is a transitive TDS in $\mathcal{M}^{\perp}$ and has no periodic point.

Proof. See the Appendix.

\section{$\S 4$. The General CASE}

Now we consider the question which system is disjoint from all minimal systems without the assumption of transitivity. We will show such a system must have a dense set of minimal points. First we prove that the set of recurrent points is dense. Note that for a dynamical system $(X, T), R(T)$ is the set of all recurrent points of $T$.

Proposition 4.1. Let $(X, T)$ be a TDS.

(1) If $X \perp Y$ with $(Y, S)$ minimal and nontrivial, then $R(T)$ is a dense $G_{\delta}$ set of $X$.

(2) Let $T$ be invertible, $V$ be an open set of $X$ and $Y=\operatorname{cl}\left(\bigcup_{i \in \mathbb{Z}} T^{n}(V)\right)$. If $(X, T) \perp \mathcal{M}$, then $(Y, T) \perp \mathcal{M}$.

(3) If there are transitive sub-systems $\left(X_{i}, T\right)$ of $(X, T)$ satisfying that $\bigcup_{i} X_{i}$ is dense in $X$ and $\left(X_{i}, T\right) \perp \mathcal{M}$ for each $i \in \mathbb{N}$. Then $(X, T) \perp \mathcal{M}$.

(4) Let $(X, T)$ be an equicontinuous system. If $(X, T) \in \mathcal{M}^{\perp}$, then each point of $X$ is a fixed point. Consequently, if $(Y, S)$ is $T D S$ with $(Y, S) \in \mathcal{M}^{\perp}$, then the maximal equicontinuous factor of $(Y, S)$ is consisting of fixed points.

Proof. (1) First we assume that $(X, T)$ is a homeomorphism. Let $\Omega(T)$ be the set of nonwandering points of $T$. If $\Omega(T) \neq X$, then there is $x \in X \backslash \Omega(T)$. Thus there 
is an open set $U$ of $X$ with $x \in U$ and $T^{i}(U) \cap T^{j}(U)=\emptyset$ whenever $i \neq j \in \mathbb{Z}$. Let $y \in Y$ and

$$
J=\operatorname{cl}\left(\bigcup_{n \in \mathbb{Z}} T^{n}(U) \times\left\{S^{n}(y)\right\}\right) \cup\left(X \backslash \bigcup_{i \in \mathbb{Z}} T^{n}(U)\right) \times Y .
$$

It is easy to see that $J$ is a joining between $(X, T)$ and $(Y, S)$ and thus $J=X \times Y$. As $Y$ is not trivial, $Y \backslash\{y\}$ is nonempty and open. As $U \times(Y \backslash\{y\}) \subset X \times Y=J$, we have

$$
U \times(Y \backslash\{y\}) \subset \operatorname{cl}\left(\bigcup_{n \in \mathbb{Z}} T^{n}(U) \times\left\{S^{n}(y)\right\}\right) .
$$

As $U \times(Y \backslash\{y\})$ is nonempty and open in $X \times Y$, there is $n \in \mathbb{Z}$ such that $(U \times(Y \backslash\{y\})) \cap\left(T^{n}(U) \times\left\{S^{n}(y)\right\}\right) \neq \emptyset$. It is clear that $n \neq 0$. Thus there is $n \neq 0$ such that $U \cap T^{n}(U) \neq \emptyset$, a contradiction. Thus we have proved $\Omega(T)=X$. By $[\mathrm{F}] R(T)$ is a dense $G_{\delta}$ set of $X$.

In the general case by Proposition $1.1(\widetilde{X}, \widetilde{T}) \perp(\widetilde{Y}, \widetilde{S})$. By the above argument we have $R(\widetilde{T})$ is dense in $\widetilde{X}$. It follows that $R(T)$ is dense in $X$. By $[\mathrm{F}] R(T)$ is a dense $G_{\delta}$ set of $X$.

(2) If $(X, T) \perp \mathcal{M}$ and $Y=X$, then $(Y, T) \perp \mathcal{M}$.

Now consider the case that $Y \neq X$. Assume that $J \subset Y \times Z$ is a joining, where $(Z, S)$ is a minimal system. Let $J^{\prime}=J \cup \operatorname{cl}(X \backslash Y) \times Z$.

As $(X, T) \perp \mathcal{M}$, we have that $J^{\prime}=X \times Z$. This implies that $J \supset V \times Z$. As $J$ is closed and invariant, we get that $J \supset\left(\bigcup_{n \in \mathbb{Z}_{+}} T^{n}(V)\right) \times Z$. Since $R(T)$ is dense in $X$, we have $T^{-n}(V) \subset \operatorname{cl}\left(\bigcup_{m \in \mathbb{Z}_{+}} T^{m}(V)\right)$ for each $n \in \mathbb{N}$. That is, $J=Y \times Z$ and hence $(Y, T) \perp \mathcal{M}$.

(3) Let $(Y, S)$ be a minimal system and $J$ is a joining between $(X, T)$ and $(Y, S)$. For each $i \in \mathbb{N}$ let $x_{i}$ be a transitive point of $\left(X_{i}, T\right)$. For each $i$ there is $y_{i}$ such that $\left(x_{i}, y_{i}\right) \in J$. Thus $J_{i}=\operatorname{cl}\left(\operatorname{orb}\left(x_{i}, y_{i}\right), T \times S\right) \subset J$. It is easy to see that $J_{i}$ is a joining between $\left(X_{i}, T\right)$ and $(Y, S)$. Thus $J_{i}=X_{i} \times Y$. This implies that $J=X \times Y$, i.e., $(X, T) \perp \mathcal{M}$.

(4) As $(X, T)$ is equicontinuous, for each $\epsilon>0$ there is $\delta>0$ such that if $d(x, y)<\delta$, then $d\left(T^{n}(x), T^{n}(y)\right)<\epsilon$ for each $n \in \mathbb{Z}$. Assume that there is $x \in X$ with $T(x) \neq x$. Let $\epsilon<d(x, T(x)) / 4$ and $\delta$ be the number corresponding to $\epsilon$ with $\delta<\epsilon$. Moreover, let $U=B(x, \delta)$ and $V=B(T(x), \delta)$.

Set $X_{1}=\operatorname{cl}\left(\bigcup_{i \in \mathbb{Z}} T^{i}(U)\right)$ and $Y_{1}=\operatorname{orb}(x, T)$. As $\left(Y_{1}, T\right)$ is minimal, $(X, T) \perp$ $\left(Y_{1}, T\right)$. Then $X_{1} \perp Y_{1}$ by (2). Let $J=\operatorname{cl}\left(\bigcup_{i \in \mathbb{Z}}\left(T^{i}(U) \times\left\{T^{i} x\right\}\right)\right.$. Then $J$ is a joining of $X_{1}$ and $Y_{1}$. Thus, $J=X_{1} \times Y_{1}$. This implies that there is $n$ with

$$
T^{n}(U) \times\left\{T^{n}(x)\right\} \cap U \times\left(V \cap Y_{1}\right) \neq \emptyset .
$$

That is, $T^{n}(U) \cap U \neq \emptyset$ and $T^{n}(x) \in V \cap Y_{1}$, a contradiction.

Now we proceed to show that if $(X, T) \in \mathcal{M}^{\perp}$, then the set of minimal points is dense in $X$. To do this we need

Definition. A sequence $\left\{F_{n}\right\}_{1}^{\infty}$ of thickly syndetic subsets of $\mathbb{Z}_{+}$is uniform, if for any $l \in \mathbb{N}$ there exists $\phi(l)$ such that for every $n \in \mathbb{N}$, we can find a subset $\left\{w_{1}^{n}(l), w_{2}^{n}(l), w_{3}^{n}(l) \cdots\right\}$ of $\mathbb{Z}_{+}$with $w_{1}^{n}(l) \leq \phi(l), w_{i+1}^{n}(l)-w_{i}^{n}(l) \leq \phi(l)$ and $\left\{w_{i}^{n}(l), w_{i}^{n}(l)+1, \cdots, w_{i}^{n}(l)+l-1\right\} \subset F_{n}, i \in \mathbb{N}$. 
Strengthening Theorem 2.3 we have

Theorem 4.2. Let $\left\{F_{n}^{\prime}\right\}_{n=1}^{+\infty}$ be a uniform sequence of thickly syndetic subsets of

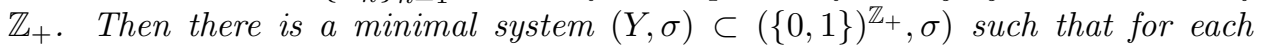
$n \in \mathbb{N}$ there exists $y_{n} \in Y$ with $N\left(y_{n},[1]\right) \subset F_{n}^{\prime}$, where $[1]=\{y \in Y: y(0)=1\}$.

Proof. We will modify the construction in Theorem 2.3. Let $f: \mathbb{N} \longrightarrow \mathbb{N}$ be a map such that for each $i \in \mathbb{N}, f^{-1}(i)$ is infinite. Let $F_{n}=F_{f(n)}^{\prime}$ for each $n \in \mathbb{N}$. Then $\left\{F_{n}\right\}$ is a uniform sequence of thickly syndetic sets.

First we modify the construction of $y^{1}$ in step 1 in Theorem 2.3 to obtain $y_{n}^{1}$ for each $n \in \mathbb{N}$, namely we construct $A_{1}$ such that $A_{1}$ appears in $y_{n}^{1}=1_{F_{n}^{1}}$ with gaps bounded by $l_{1}$ and $F_{n}^{1} \subset F_{n}$.

Take $k_{1} \in \mathbb{N}$ and $A_{1}=0 \ldots 01$ with $\left|A_{1}\right|=k_{1}$. Set $r_{1}=k_{1}$. As $\left\{F_{n}\right\}$ is a uniform sequence of thickly syndetic sets, $1^{r_{1}}$ appears in $F_{n}$ at a syndetic set $\left\{w_{1}^{n}\left(r_{1}\right), w_{2}^{n}\left(r_{1}\right), \ldots\right\}$. Without loss of generality, assume that $w_{1}^{n}\left(r_{1}\right) \leq l_{1}-k_{1}$ and $2 r_{1} \leq w_{j+1}^{n}-w_{j}^{n} \leq l_{1}$, where $l_{1}$ is some number in $\mathbb{N}$. Choose $y_{n}^{1} \in\{0,1\}^{\mathbb{Z}_{+}}$such that

$y_{n}^{1}\left[w_{i}^{n}\left(r_{1}\right) ; w_{i}^{n}\left(r_{1}\right)+k_{1}-1\right]=A_{1}$ and

$y_{n}^{1}(j)=0$ if $j \in \mathbb{Z}_{+} \backslash\left(\bigcup_{i=1}^{\infty}\left[w_{i}^{n}\left(r_{1}\right) ; w_{i}^{n}\left(r_{1}\right)+k_{1}-1\right]\right)$.

It is easy to see that for each $n \in \mathbb{N}, A_{1}$ appears in $y_{n}^{1}$ with gaps bounded by $l_{1}$ and $F_{n}^{1} \subset F_{n}$, where $1_{F_{n}^{1}}=y_{n}^{1}$.

Let $A_{2}=A_{1} y_{1}^{1}\left[0 ; a_{1}\right] A_{1}$ with $a_{1}>\phi\left(k_{1}\right)$ and $y_{1}^{1}\left[0 ; a_{1}\right]=A_{1}^{\prime} A_{1}$. It is easy to see that $A_{2}\left[k_{1} ; k_{1}+a_{1}\right] \subset F_{1}$.

Set $k_{2}=\left|A_{2}\right|$. Now we modify the construction of step 2 in Theorem 2.3 as we did (for step 1) to obtain $y_{n}^{2}$ (as $1^{k_{2}}$ appears in $F_{n}$ syndetically). Then $A_{1}, A_{2}$ appear in $y_{n}^{2}$ syndetically with gaps bounded by $2 l_{1}$ and $l_{2}$ respectively. Set $A_{3}=A_{2} y_{2}^{2}\left[0, a_{2}\right] A_{2}$ with $a_{2}>\phi\left(k_{2}\right)$ and $y_{2}^{2}\left[0 ; a_{2}\right]=A_{2}^{\prime} A_{2}$. It is easy to see that $A_{3}\left[k_{2}, k_{2}+a_{2}\right] \subset F_{2}$.

By the same arguments, we get $y_{n}^{i}$ and we set $A_{i+1}=A_{i} y_{i}^{i}\left[0, a_{i}\right] A_{i}$ with $a_{i}>$ $\phi\left(k_{i}\right)$ and $y_{i}^{i}\left[0 ; a_{i}\right]=A_{i}^{\prime} A_{i}$. We have $A_{i+1}\left[k_{i}, k_{i}+a_{i}\right] \subset F_{i}$. Moreover, $A_{1}, \ldots, A_{i}$ appear in $y_{n}^{i}$ syndetically with gaps bounded by $2 l_{1}, \ldots, 2 l_{i-1}, l_{i}$ respectively for each $n$.

Let $y=\lim A_{i}$. Then $y$ is a minimal point. For each $n$ let $y_{n}$ be a limit point of $\left\{A_{i+1}\left[k_{i}, k_{i}+a_{i}\right]\right\}_{f(i)=n}$. Then we have $N\left(y_{n},[1]\right) \subset F_{n}^{\prime}$.

With the help of Theorem 4.2 we now show

Theorem 4.3. Let $(X, T)$ be a dynamical system. If $(X, T)$ is in $\mathcal{M}^{\perp}$, then $(X, T)$ has a dense set of minimal points.

Proof. First assume that $T$ is a homeomorphism and that the set of minimal points $M$ is not dense in $X$. Then there are an open set $U$ of $X$ and an open neighborhood $V$ of $c l(M)$ with $U \cap V=\emptyset$.

By Theorem 4.1(1), $R(T)$ is dense in $U$. Then we may take recurrent points $\left\{x_{n}\right\}_{n=1}^{+\infty} \subset U$ such that $\left\{x_{n}: n \in \mathbb{N}\right\}$ is dense in $U$. Let $F_{n}=N\left(x_{n}, U^{c}\right) \supset$ $N\left(x_{n}, V\right), n=1,2, \cdots$. We claim that

$\left\{F_{n}\right\}_{n=1}^{+\infty}$ is a uniform sequence of thickly syndetic subsets of $\mathbb{Z}_{+}$.

Proof of claim. For any $l>0$, there exists a neighborhood $W_{l} \subset V$ of $\operatorname{cl}(M)$ such that $T^{k}\left(W_{l}\right) \subset V$ for each $1 \leq k \leq l$. As $\bigcup_{i \in \mathbb{Z}_{+}} T^{-i} W_{l}=X$ and $X$ is compact, there exists $\phi(l)>0$ such that $\bigcup_{0 \leq i \leq \phi(l)} T^{-i} W_{l}=X$. 
For each $n \in \mathbb{N}$, let $N\left(x_{n}, W_{l}\right)=\left\{w_{1}^{n}, w_{2}^{n}, \cdots\right\}$. It is easy to see that $w_{1}^{n} \leq \phi(l)$, $w_{i+1}^{n}-w_{i}^{n} \leq \phi(l)$ and $\left\{w_{i}^{n}, w_{i}^{n}+1, \cdots, w_{i}^{n}+l-1\right\} \subset N\left(x_{n}, V\right)$. As $F_{n} \supset N\left(x_{n}, V\right)$, this shows that $\left\{F_{n}\right\}_{n=1}^{+\infty}$ is a uniform sequence of thickly syndetic subsets of $\mathbb{Z}_{+}$.

By the above claim and Theorem 4.2, there is a minimal system $(Y, \sigma) \subset$ $\left(\{0,1\}^{\mathbb{Z}_{+}}, \sigma\right)$ such that for each $n \in \mathbb{N}$ there exists $y_{n} \in Y$ with $N\left(y_{n},[1]\right) \subset F_{n}$, where $[1]=\{y \in Y: y(0)=1\}$. Let

$$
J=\operatorname{cl}\left(\bigcup_{i=0}^{+\infty} \bigcup_{n=1}^{+\infty}(T \times \sigma)^{i}\left(x_{n}, y_{n}\right)\right) \cup\left(X \backslash \bigcup_{i=-\infty}^{+\infty} T^{i} U\right) \times Y .
$$

As $\left\{x_{n}: n \in \mathbb{N}\right\}$ is dense in $U$ and each $x_{n}$ is recurrent, we have

$$
\bigcup_{i=-\infty}^{+\infty} T^{i} U \subset \operatorname{cl}\left(\bigcup_{i=0}^{+\infty} \bigcup_{n=1}^{+\infty} T^{i} x_{n}\right)
$$

Thus $J$ is a joining of $X$ and $Y$, and hence $J=X \times Y$.

It is easy to see that $\operatorname{cl}\left(\bigcup_{i=0}^{+\infty} \bigcup_{n=1}^{+\infty}(T \times \sigma)^{i}\left(x_{n}, y_{n}\right)\right) \supset U \times[1]$. As $U \times[1]$ is open, we have

$$
\left(\bigcup_{i=0}^{+\infty} \bigcup_{n=1}^{+\infty}(T \times \sigma)^{i}\left(x_{n}, y_{n}\right)\right) \cap(U \times[1]) \neq \emptyset
$$

Therefore, there exist $i$ and $n$ with $\left(T^{i} x_{n}, \sigma^{i} y_{n}\right) \in U \times[1]$, i.e., $N\left(x_{n}, U\right) \cap$ $N\left(y_{n},[1]\right) \neq \emptyset$, which contradicts the fact that $N\left(y_{n},[1]\right) \subset F_{n}=N\left(x_{n}, U^{c}\right)$.

In the general case we pass to the natural extension and observe that minimal points are mapped to minimal points by factor maps.

For a dynamical system $(X, T)$, a transitive subsystem $(Y, T)$ is maximal if it is maximal among all transitive subsystems by the inclusion. We have

Theorem 4.4. Let $(X, T)$ be a TDS and $(Y, T)$ be a transitive subsystem. Then there is a MTSS containing $(Y, T)$. Consequently, if $R(T)=X$, then the union of all MTSS is dense in $X$.

Proof. Let $\mathcal{A}$ be the collection of all transitive subsystems containing $Y$. By Hausdorff maximal principal (see, for example, [K, p. 32]) there is a maximal nest $\mathcal{A}^{\prime}$ containing $Y$. It is easy to see that $\operatorname{cl}\left(\bigcup_{Z \in \mathcal{A}^{\prime}} Z\right)$ is transitive and maximal.

Inspired by the above theorem and Theorem 4.1 one may have

Conjecture. If $(X, T) \perp \mathcal{M}$, then there are countably many MTSS such that their union is dense in $X$ and each of them is in $\mathcal{M}^{\perp}$.

Unfortunately the conjecture is not true in general. However, we will show that it is true in a certain sense, i.e., if $(X, T) \perp(Y, S)$ with $Y$ nontrivial minimal, then there are countably many MTSS such that their union is dense in $X$ and each of them is disjoint from $Y$. We also give an example to show that this is the best situation we can expect, namely there are distal systems in $\mathcal{M}^{\perp}$.

Theorem 4.5. Let $(X, T)$ be a TDS and $(Y, S)$ be nontrivial minimal system. Then $(X, T) \perp(Y, S)$ if and only if there exist countably many MTSS such that their union is dense in $X$ and each of them is disjoint from $Y$. 
Proof. The sufficiency is easy and it remains to show the necessity. First we assume that $T$ and $S$ are homeomorphisms.

Let $(X, T) \perp(Y, S)$. For any open subsets $V$ of $Y$ and $W \subset X$, put

$$
\begin{aligned}
& X(U, V)=\left\{x \in X: N_{\mathbb{Z}}(x, U) \cap N_{\mathbb{Z}}(y, V) \neq \emptyset \text { for any } y \in Y\right\}, \\
& \widetilde{X}(U, V)=\left(X \backslash \operatorname{cl}\left(\bigcup_{n \in \mathbb{Z}} T^{n} U\right)\right) \cup X(U, V),
\end{aligned}
$$

where $N_{\mathbb{Z}}(x, U)=\left\{n \in \mathbb{Z}: T^{n} x \in U\right\}$ and $N_{\mathbb{Z}}(y, V)=\left\{n \in \mathbb{Z}: S^{n} y \in V\right\}$.

As $N_{\mathbb{Z}}(T x, U)=N_{\mathbb{Z}}(x, U)-1$ and $N_{\mathbb{Z}}(S y, V)=N_{\mathbb{Z}}(y, V)-1$, it is not difficult to see that $T X(W, V)=X(W, V)$. We have

Claim. For any open set $U$ of $X$ and open set $V$ of $Y$, the invariant set $\tilde{X}(U, V)$ is a dense open subset of $X$.

Proof of Claim. First, we show that $\tilde{X}(U, V)$ is an open subset of $X$. Since $X \backslash$ $\left.\operatorname{cl}\left(\bigcup_{n \in \mathbb{Z}} T^{n} U\right)\right)$ is open, it remains to show that $X(U, V)$ is open. Let $x_{n} \notin X \backslash$ $X(U, V)$ and $\lim _{n \rightarrow \infty} x_{n}=x$. Then for each $n \in \mathbb{N}$ there exists $y_{n} \in Y$ such that $N_{\mathbb{Z}}\left(x_{n}, U\right) \cap N_{\mathbb{Z}}\left(y_{n}, V\right)=\emptyset$, i.e., $\left(x_{n}, y_{n}\right) \notin \bigcup_{m \in \mathbb{Z}}(T \times S)^{m} U \times V$. Without loss of generality, assume $\lim _{n \rightarrow \infty} y_{n}=y$. Since $\bigcup_{m \in \mathbb{Z}}(T \times S)^{m} U \times V$ is open, one has $(x, y) \notin \bigcup_{m \in \mathbb{Z}}(T \times S)^{m} U \times V$, i.e., $N_{\mathbb{Z}}(x, U) \cap N_{\mathbb{Z}}(y, V)=\emptyset$. Hence $x \notin X \backslash X(U, V)$. This shows that $X \backslash X(U, V)$ is a closed set, i.e., $X(U, V)$ is an open set.

Next we show that $\tilde{X}(U, V)$ is dense in $X$. Let $W \subset U$ be an open subset of $X$. If $X(W, V) \cap W=\emptyset$, then for each $x \in W$ there exists $y(x) \in Y$ such that $N_{\mathbb{Z}}(x, W) \cap N_{\mathbb{Z}}(y(x), V)=\emptyset$.

Let

$$
\left.J=\left(X \backslash\left(\bigcup_{n \in \mathbb{Z}} T^{n} W\right)\right) \times Y\right) \cup \operatorname{cl}\left(\bigcup_{n \in \mathbb{Z}} \bigcup_{x \in W}\left(T^{n} \times S^{n}\right)(x, y(x))\right) .
$$

Clearly, $J$ is a joining of $X$ and $Y$. As $N_{\mathbb{Z}}(x, W) \cap N_{\mathbb{Z}}(y(x), V)=\emptyset$ for each $x \in W$, one has

$$
\operatorname{cl}\left(\bigcup_{n \in \mathbb{Z}} \bigcup_{x \in W}\left(T^{n} \times S^{n}\right)(x, y(x))\right) \cap W \times V=\emptyset .
$$

Therefore, $J \cap W \times V=\emptyset$. Hence $J \neq X \times Y$ which contradicts $(X, T) \perp(Y, S)$. This shows that $X(W, V) \cap W \neq \emptyset$ and thus $X(U, V) \cap W \neq \emptyset$. As $W$ is arbitrary and $X(U, V)$ is $T$-invariant, one has $\operatorname{cl}(X(U, V)) \supset \operatorname{cl}\left(\bigcup_{n \in \mathbb{Z}} T^{n} U\right)$. Therefore, $\widetilde{X}(U, V)$ is dense in $X$. This ends the proof of claim.

Let $\left\{V_{n}\right\}_{n=1}^{+\infty}$ and $\left\{U_{m}\right\}_{m=1}^{+\infty}$ be basis of $Y$ and $X$ respectively. Put

$$
R_{X}=\bigcap_{m=1}^{\infty} \bigcap_{j=1}^{\infty} \tilde{X}\left(U_{m}, V_{j}\right)
$$

As $\widetilde{X}\left(U_{m}, V_{j}\right)$ is a dense open invariant subset of $X$ for $m, j \in \mathbb{N}$ by the claim, $R_{X}$ is a dense $G_{\delta}$ set of $X$. Now we use a terminology given by Auslander: A set $A \subset X$ has the capturing property if $\omega(p, T) \cap A \neq \emptyset$ implies $p \in A$. It is easy to see that any open invariant subset of $X$ has the capturing property and any intersection of capturing sets is capturing. In particular, $R_{X}$ has the capturing property.

For each $x \in R_{X} \cap R(T)$, set $X_{x}=\operatorname{cl}(\operatorname{orb}(x, T))$. Then $\left(X_{x}, T\right)$ is a transitive subsystem. Hence by Theorem 4.4 there exists $x^{\prime} \in R(T)$ such that $\left(X_{x^{\prime}}, T\right)$ is a 
maximal transitive subsystem and $X_{x^{\prime}} \supset X_{x}$, where $X_{x^{\prime}}=\operatorname{cl}\left(\operatorname{orb}\left(x^{\prime}, T\right)\right)$. Since $R_{X}$ has the capturing property and $x \in X_{x^{\prime}} \cap R_{X}$, one gets $x^{\prime} \in R_{X}$.

Now, we show that $\left(X_{x^{\prime}}, T\right) \perp(Y, S)$. Let $J$ be a joining of $X_{x^{\prime}}$ and $Y$, and $J^{\prime}=\bigcap_{n \in \mathbb{Z}}(T \times S)^{n} J$. Then $J^{\prime}$ is a joining with $T \times S\left(J^{\prime}\right)=J^{\prime}$. Take $y \in Y$ such that $\left(x^{\prime}, y\right) \in J^{\prime}$.

For any open neighborhood $U$ of $x^{\prime}$ and open set $V$ of $Y$, there exist $U_{m}, V_{j}$ such that $x \in U_{m} \subset U$ and $V_{j} \subset V$. By (4.5.1), $x^{\prime} \in X\left(U_{m}, V_{j}\right)$. In particular, $N_{\mathbb{Z}}\left(x^{\prime}, U_{m}\right) \cap N_{\mathbb{Z}}\left(y, V_{j}\right) \neq \emptyset$. As $T \times S\left(J^{\prime}\right)=J^{\prime}$ and $\left(x^{\prime}, y\right) \in J^{\prime}, J^{\prime} \cap U_{m} \times V_{j} \neq \emptyset$. Thus $J^{\prime} \supset\left\{x^{\prime}\right\} \times Y$ and hence $J^{\prime}=X_{x^{\prime}} \times Y$. Therefore, $\left(X_{x^{\prime}}, T\right) \perp(Y, S)$.

By Proposition 4.1(1), $R(T)$ is a dense $G_{\delta}$ set of $X$. Moreover, $R_{X} \cap R(T)$ is dense in $X$. As $\bigcup_{x \in R_{X} \cap R(T)} X_{x^{\prime}}$ is dense in $X$, we can find countably many MTSS such that their union is dense in $X$ and each of them is disjoint from $Y$.

In the general case we pass to the natural extension. Let $\widetilde{X}$ and $\widetilde{Y}$ be natural extensions of $X$ and $Y$ respectively. Then by Proposition 1.1, $\widetilde{X} \perp \widetilde{Y}$. Thus there exist countably many MTSS $\widetilde{X}_{i}$ of $\widetilde{X}$ such that their union is dense in $\widetilde{X}$ and each of them is disjoint from $\tilde{Y}$. Let $\pi: \widetilde{X} \longrightarrow X$ be the projection to the first coordinate. Then $\pi\left(\widetilde{X}_{i}\right)$ are MTSS of $X$ and their union is dense in $X$. It is clear that $\widetilde{X}_{i} \perp Y$.

Using Theorem 4.5, we get easily

Corollary 4.6. Let $(X, T)$ be a dynamical system. Then $(X, T) \perp \mathcal{M}$ iff for any minimal system $(Y, S)$ there exist countably many MTSS (depending on $Y$ ) such that their union is dense in $X$ and each of them is disjoint from $Y$.

To finish the section we now construct the example we promised. We need

Definition. Let $(X, T)$ be a TDS and $f$ a complex-valued continuous function on $X$ which is not identically 0 . We say that $f$ is an eigenfunction for $T$ if there exists $\lambda \in \mathbb{C}$ such that $f(T x)=\lambda f(x)$ for any $x \in X$. We then call $\lambda$ the eigenvalue for $T$ corresponding to the eigenfunction $f$. We denote the set of eigenvalues of $T$ by $\operatorname{Eig}(T)$.

We will give an equivalence condition for a distal system in $\mathcal{M}^{\perp}$. To do so we need Lemma 4.7 and 4.8 .

Lemma 4.7 (Wa $)$. Let $(X, T)$ be a transitive TDS and $T$ a homeomorphism. Then Eig $(T)$ forms a countable subgroup of $K$, where $K$ is the unit circle in complex plane.

If $T: K \longrightarrow K$ is defined by $T(z)=e^{i 2 \pi \theta} z$, where $\theta$ is irrational, then $\operatorname{Eig}(T)=$ $\left\{e^{i 2 \pi n \theta}: n \in \mathbb{Z}\right\}$.

Lemma 4.8. Let $(X, T)$ and $(Y, S)$ be minimal TDS.

(1) If $(X, T)$ is distal and $\left(Y_{\mathrm{eq}}, S\right)$ is the maximal equicontinous factor of $(Y, S)$, then $(X, T) \perp(Y, S)$ if and only if $(X, T) \perp\left(Y_{\mathrm{eq}}, S\right)$.

(2) If $(X, T)$ is equicontinuous, then $(X, T) \perp(Y, S)$ iff $X$ and $Y$ have no nontrivial common factor.

(3) If $(X, T)$ is equicontinuous and $\operatorname{Eig}(T) \cap \operatorname{Eig}(S)=\{1\}$, then $(X, T) \perp$ $(Y, S)$.

Proof. For the proofs of (1) and (2), see [A]. Now we show (3). By (2), it remains to show that $X$ and $Y$ have no nontrivial common factor. 
Assume that $X$ and $Y$ have nontrivial common factor $(Z, H)$. Then $(Z, H)$ is a minimal equicontinuous system, and there exists $\lambda \in \operatorname{Eig}(H) \backslash\{1\}$. As $\operatorname{Eig}(H) \subset$ $\operatorname{Eig}(T) \cap \operatorname{Eig}(S), \lambda \in \operatorname{Eig}(T) \cap \operatorname{Eig}(S)$, a contradiction.

Theorem 4.9. Let $(X, T)$ be a distal system. Then $(X, T) \perp \mathcal{M}$ iff $(X, T)$ is disjoint from any minimal equicontinuous system.

Proof. It remains to show that if $(X, T)$ is disjoint from any minimal equicontinuous system, then $(X, T) \perp \mathcal{M}$. Let $(Y, S)$ be a minimal system and let $\left(Y_{\text {eq }}, S\right)$ be its maximal equicontinuous factor. If $Y_{\text {eq }}$ is trivial, then $(Y, S)$ is minimal and weakly mixing. By Theorem 1.3, $(X, T) \perp(Y, S)$.

Now, assume that $Y_{\text {eq }}$ is nontrivial. Since $(X, T) \perp\left(Y_{\text {eq }}, S\right)$, by Corollary 4.6 there exist transitive subsystems $\left(X_{i}, T\right)$ of $(X, T)$ satisfying $\bigcup_{i} X_{i}$ is dense in $X$ and $\left(X_{i}, T\right) \perp\left(Y_{\text {eq }}, S\right)$ for each $i$. As $\left(X_{i}, T\right)$ is distal and transitive, $\left(X_{i}, T\right)$ is minimal and distal. By Lemma 4.8(1), one has $\left(X_{i}, T\right) \perp(Y, S)$. Hence $(X, T) \perp(Y, S)$. This implies that $(X, T) \perp \mathcal{M}$.

Finally, we can construct the following example.

Example 4.10. Let $X=[0,1] \times K$ and define $T: X \longrightarrow X$ by $T(x, z)=\left(x, e^{i 2 \pi x} z\right)$. Then $(X, T)$ is distal and $(X, T) \perp \mathcal{M}$.

Proof. Clearly, $(X, T)$ is distal. By Theorem 4.9 it remains to show $(X, T)$ is disjoint from any minimal equicontinuous system. Let $(Y, S)$ be a minimal equicontinuous system. Then $\operatorname{Eig}(S)$ is a countable set of $K$. Set $E_{X}=\left\{x \in[0,1] \backslash \mathbb{Q}: e^{i 2 \pi n x} \notin\right.$ $\operatorname{Eig}(S)$ for each $n \in \mathbb{Z} \backslash\{0\}\}$. As $\operatorname{Eig}(S)$ is countable, it is easy to see that $E_{X}$ is dense in $[0,1]$.

For each $x \in E_{X}$, let $X_{x}=\{x\} \times K . X_{x}$ can be considered as an irrational rotation of $K$. By Lemma 4.7, $\operatorname{Eig}\left(X_{x}, T\right)=\left\{e^{i 2 \pi n x}: n \in \mathbb{Z}\right\}$. From the definition of $E_{X},\left\{e^{i 2 \pi n x}: n \in \mathbb{Z}\right\} \cap \operatorname{Eig}(S)=\{1\}$. By Lemma $4.8(3),\left(X_{x}, T\right) \perp(Y, S)$. This shows that $(X, T)$ is disjoint from any minimal equicontinuous system by Proposition 4.1(3). Thus $(X, T) \perp \mathcal{M}$.

\section{§5. An EXTREMELY SCATTERING, NONWEAKLY MIXING EXAMPLE}

In this section we will construct an extremely scattering, nonweakly mixing example. To do this we need some lemmas to check when a system is not weakly mixing and is extremely scattering. Roughly speaking, the reason such an example exists is that not all syndetic sets can be realized by a dynamical system (Lemma $5.2)$.

Recall that for a dynamical system $(X, T), x \in X$, and a pair of nonempty subsets $U, V$ of $X, N(x, U)=\left\{n \in \mathbb{Z}_{+}: T^{n}(x) \in U\right\}$ and $N(U, V)=\left\{n \in \mathbb{Z}_{+}\right.$: $\left.U \cap T^{-n} V \neq \emptyset\right\}$. The following lemma will be used in the construction and also can be viewed as another characterization of weak mixing.

Lemma 5.1. Let $(X, T)$ be a transitive TDS. If for any open subset $U$ of $X$ there exists $s=s_{U} \in \mathbb{Z}_{+}$such that $s, s+1 \in N(U, U)$, then $(X, T)$ is weakly mixing.

Proof. It is known that $(X, T)$ is weakly mixing iff it is transitive and $N(U, U)$ is thick for any open $U$. Thus it remains to show $N(U, U)$ is thick for any open subset $U$ of $X$. First we have

Claim. If $N(U, U)$ contains consecutive natural numbers of length $k$, then $N(U, U)$ contains consecutive natural numbers of length $k+1$. 
Proof of Claim. Let $a, a+1, \cdots, a+k-1 \in N(U, U)$ with $a \in \mathbb{N}$. Then $U \cap$ $T^{-(a+i)} U \neq \emptyset, 0 \leq i \leq k-1$. Since $(X, T)$ is transitive, there exist $t_{0}, t_{1}, \cdots, t_{k-1} \in$ $\mathbb{N}$ (by induction) such that

$$
\bigcap_{i=0}^{k-1} T^{-t_{i}}\left(U \cap T^{-(a+i)} U\right) \neq \emptyset
$$

Put $D=\bigcap_{i=0}^{k-1} T^{-t_{i}}\left(U \cap T^{-(a+i)} U\right)$. By the assumption there is $s \in \mathbb{Z}_{+}$such that $s, s+1 \in N(D, D)$, i.e., $D \cap T^{-s} D \neq \emptyset$ and $D \cap T^{-(s+1)} D \neq \emptyset$. Clearly,

$$
\begin{array}{r}
\left(U \cap T^{-(a+i)} U\right) \cap T^{-s}\left(U \cap T^{-(a+i)} U\right) \neq \emptyset, \\
\left(U \cap T^{-(a+i)} U\right) \cap T^{-(s+1)}\left(U \cap T^{-(a+i)} U\right) \neq \emptyset,
\end{array}
$$

for $i=0,1, \cdots, k-1$.

Particularly, $U \cap T^{-(s+a+i)} U \neq \emptyset$ and $U \cap T^{-(s+1+a+i)} U \neq \emptyset$ for $0 \leq i \leq k-1$. Hence, one has $s+a, s+a+1, \cdots, s+a+k-1, s+a+k \in N(U, U)$. This finishes the proof of the claim.

By the claim, $N(U, U)$ is thick for any open subset $U$ of $X$.

Lemma 5.2. Let $(X, T)$ be a topologically ergodic system. Then for any open subset $U$ of $X$ and $k \in \mathbb{N}, N_{k}(U)=\left\{i \in \mathbb{Z}_{+}: 2^{k} i \in N(U, U)\right\}$ is syndetic.

Proof. First, we consider the case when $k=1$. If $N(U, U)$ doesn't contain odd numbers, clearly $N_{1}(U)$ is syndetic. Assume now there exists an odd number $a_{1} \in$ $N(U, U)$, that is, $U \cap T^{-a_{1}} U \neq \emptyset$. Put $D_{1}=U \cap T^{-a_{1}} U$. Take any $m \in N\left(D_{1}, D_{1}\right)$, then $D_{1} \cap T^{-m} D_{1} \neq \emptyset$. This implies $U \cap T^{-m} U \cap T^{-\left(m+a_{1}\right)} U \neq \emptyset$. Thus, $\left\{m, a_{1}+\right.$ $m\} \subset N(U, U)$. Since $a_{1}$ is odd and $N\left(D_{1}, D_{1}\right)$ is syndetic, it turns out that $N_{1}(U)$ is syndetic.

Assume that for any $1 \leq k \leq l, N_{k}(U)$ is syndetic for any open subset $U$ of $X$. We shall show that $N_{l+1}(U)$ is syndetic for any open subset $U$ of $X$.

If $N_{l}(U)$ doesn't contain odd numbers, then $N_{l+1}(U)$ is syndetic. Assume now there is an odd number $a_{l} \in N_{l}(U)$, i.e., $D_{l}=U \cap T^{-2^{l} a_{l}} U \neq \emptyset$. Similarly, one has $2^{l} m, 2^{l}\left(m+a_{l}\right) \subset N(U, U)$ for each $m \in N_{l}\left(D_{l}\right)$. This implies that $m, m+a_{l} \in N_{l}(U)$ for each $m \in N_{l}\left(D_{l}\right)$. Since $a_{l}$ is odd and $N_{l}\left(D_{l}\right)$ is syndetic (by the inductive assumption), $N_{l+1}(U)$ is syndetic.

Lemma 5.3. Let $(X, T)$ be a transitive system with a transitive point $x$. If for any neighborhood $U$ of $x$ we have property $(* *)$ :

$(* *)$ for any $r \in \mathbb{Z}_{+}$there exists $k_{r} \in \mathbb{N}$ such that $N_{k_{r}}(U, r)=\left\{n \in \mathbb{N}: 2^{k_{r}} n-r \in\right.$ $N(U, U)\}$ is thick,

then $(X, T)$ is extremely scattering.

Proof. First let $(Y, S)$ be invertible and topologically ergodic. Let $U_{1}, U_{2}$ be any open subsets of $X$ and let $V_{1}, V_{2}$ be any open subsets of $Y$. Then by the definition

$$
N\left(U_{1} \times V_{1}, U_{2} \times V_{2}\right)=\left\{n \in \mathbb{Z}_{+}:(T \times S)^{-n}\left(U_{2} \times V_{2}\right) \cap\left(U_{1} \times V_{1}\right) \neq \emptyset\right\} .
$$


As $x$ is a transitive point, there are $n_{0}, k$ such that $U=T^{-\left(n_{0}+k\right)}\left(U_{2}\right) \cap T^{-k}\left(U_{1}\right)$ is a nonempty neighborhood of $x$. Thus,

$$
\begin{aligned}
& N\left(U_{1} \times V_{1}, U_{2} \times V_{2}\right) \\
& =\left\{n \in \mathbb{Z}_{+}:(T \times S)^{-(n+k)}\left(U_{2} \times V_{2}\right) \cap(T \times S)^{-k}\left(U_{1} \times V_{1}\right) \neq \emptyset\right\} \\
& =\left\{n \in \mathbb{Z}_{+}:\left(T^{-(n+k)} U_{2} \cap T^{-k} U_{1}\right) \times\left(S^{-(n+k)} V_{2} \cap S^{-k} V_{1}\right) \neq \emptyset\right\} \\
& \supset n_{0}+\left\{m \in \mathbb{Z}_{+}:\left(T^{-m} U \cap U\right) \times\left(S^{-m} S^{-\left(n_{0}+k\right)} V_{2} \cap S^{-k} V_{1}\right) \neq \emptyset\right\} .
\end{aligned}
$$

Since $(Y, S)$ is transitive and $S$ is a homeomorphism, there exist an open subset $V \in S^{-k}\left(V_{1}\right)$ and $r \in \mathbb{N}$ such that $S^{-r} V \subset S^{-\left(n_{0}+k\right)}\left(V_{2}\right)$. Thus

$$
N\left(U_{1} \times V_{1}, U_{2} \times V_{2}\right) \supset n_{0}+N\left(U \times V, U \times S^{-r} V\right) .
$$

By property $(* *)$ there exists $k_{r}$ such that $N_{k_{r}}(U, r)$ is thick. By Lemma 5.2, one knows that $N_{k_{r}}(V)$ is syndetic.

Thus $N_{k_{r}}(U, r) \cap N_{k_{r}}(V) \neq \emptyset$. Let $m \in N_{k_{r}}(U, r) \cap N_{k_{r}}(V)$. Then $2^{k_{r}} m-r \in$ $N(U, U)$ and $2^{k_{r}} m \in N(V, V)$, and thus we have

$$
2^{k_{r}} m-r \in N\left(V, S^{-r} V\right) \cap N(U, U)=N\left(U \times V, U \times S^{-r} V\right) .
$$

Hence $n_{0}+2^{k_{r}} m-r \in N\left(U_{1} \times V_{1}, U_{2} \times V_{2}\right)$.

Now assume that $(Y, S)$ is a topologically ergodic system and $\left(Y_{1}, S_{1}\right)$ is the nature extension $(Y, T)$. Then $\left(Y_{1}, S_{1}\right)$ is invertible and topologically ergodic. Hence by what we have proved, $\left(X \times Y_{1}, T \times S_{1}\right)$ is transitive. As $(X \times Y, T \times S)$ is a factor of $\left(X \times Y_{1}, T \times S_{1}\right),(X \times Y, T \times S)$ is transitive, i.e., $(X, T)$ is extremely scattering. This ends the proof.

With the above preparation we now start to construct the example we promised.

Theorem 5.4. There is an extremely scattering, not weakly mixing system.

Proof. We will construct the system in a one sided shift on two symbols $(\Sigma, S)$ and the system is the closure of the orbit of a recurrent point $x=\left(x_{0}, x_{1}, \cdots\right) \in \Sigma$. To do this, we construct inductively a sequence of finite words $C_{i}$ such that $C_{i+1}$ begins with $C_{i}$ and $x$ is just the limit of $C_{i}$.

Define $F: \mathbb{N} \longrightarrow \mathbb{N} \times \mathbb{Z}_{+}$by $F(l)=(\phi(l), \varphi(l))$ such that $\phi(l+1) \leq l$ and for any $(n, r) \in \mathbb{N} \times \mathbb{Z}_{+}$there exist infinitely many $j \in \mathbb{N}$ with $F(j)=(n, r)$. To begin with we let

$$
C_{0}=(0), C_{1}=(0,0,1,0,0)=\left(x_{0}, x_{1}, \cdots, x_{4}\right) \text { and } k_{1}=5 .
$$

Set

$$
W_{1}=\{2\}=\left\{i: x_{i}=1, i \leq k_{1}-1\right\} \text { and } B_{1}=W_{1}-W_{1}=\{0\} .
$$

where $A-B=\{a-b \geq 0: a \in A, b \in B\}$. Inductively we construct $C_{l}$. If $k_{l}$ is the length of $C_{l}$, then we define

$$
W_{l}=\left\{i: x_{i}=1,0 \leq i \leq k_{l}-1\right\} \text { and } B_{l}=W_{l}-W_{l} .
$$

Moreover, $B_{l}$ satisfies

$(1)_{l} 1 \notin B_{l}$ and there is no $s \in \mathbb{Z}_{+}$such that $\{s, s+1\} \subset B_{l}$.

(2) $C_{l}$ begins with $C_{l-1}$. 
For $l=1,(1)_{l}$ and $(2)_{l}$ are satisfied. Assume that we have constructed $C_{n}$ for $1 \leq n \leq l$ with $(1)_{n}$ and $(2)_{n}$. We build $C_{l+1}$ as follows.

Assume that $p_{l, 1}, p_{l, 2}, \cdots, p_{l, l+1}, q_{l}$ are positive integers to be defined later and set

$$
q_{l, i}=2^{k_{\phi(l+1)}+1}\left(q_{l}+i\right)-k_{\phi(l+1)}-\varphi(l+1), \text { for } i=1,2, \cdots, l+1 .
$$

Let

$$
\begin{aligned}
C_{l+1}= & C_{l} 0^{p_{l, 1}} C_{\phi(l+1)} 0^{q_{l, 1}} C_{\phi(l+1)} 0^{p_{l, 2}} C_{\phi(l+1)} 0^{q_{l, 2}} C_{\phi(l+1)} 0^{p_{l, 3}} \cdots \\
& 0^{p_{l, l+1}} C_{\phi(l+1)} 0^{q_{l, l+1}} C_{\phi(l+1)} .
\end{aligned}
$$

Set

$$
a_{1}^{l}=k_{l}+p_{l, 1}, a_{2 i-1}^{l}=k_{\phi(l+1)}+p_{l, i} \text { and } a_{2 j}^{l}=q_{l, j}+k_{\phi(l+1)},
$$

where $i=2,3, \cdots, l+1$ and $j=1,2, \cdots, l+1$.

By (5.1) and (5.4)

$$
W_{l+1}=W_{l} \cup \bigcup_{k=1}^{2(l+1)}\left(W_{\phi(l+1)}+\left(a_{1}^{l}+a_{2}^{l}+\cdots+a_{k}^{l}\right)\right) .
$$

As $W_{\phi(l+1)} \subset W_{l}$, we know that $W_{l}-W_{\phi(l+1)}, W_{\phi(l+1)}-W_{l} \subset W_{l}-W_{l}=B_{l}$. Thus from (5.1) and (5.5) we have

$$
\begin{aligned}
B_{l+1} \subset B_{l} & \cup \bigcup_{k=1}^{2(l+1)}\left(\left(a_{1}^{l}+a_{2}^{l}+\cdots+a_{k}^{l}\right) \pm B_{l}\right) \\
& \cup \bigcup_{1 \leq i<j \leq 2(l+1)}\left(\left(a_{i+1}^{l}+a_{i+2}^{l}+\cdots+a_{j}^{l}\right) \pm B_{\phi(l+1)}\right) .
\end{aligned}
$$

We can take $p_{l, 1}, p_{l, 2}, \cdots, p_{l, l+1}, q_{l}$ such that $B_{l+1}$ satisfies $(1)_{l+1}$. We do this at the end of the proof.

Let $x=\lim _{l} C_{l}$ and $X$ be the orbit closure of $x$ under the shift $S$. We now prove that $(X, S)$ is extremely scattering and not weakly mixing.

Let $U=\left\{y \in X: y_{0}=1\right\}$. Then

$$
N(x, U)=\bigcup_{l=1}^{+\infty} W_{l} \text { and } N(U, U)=\bigcup_{l=1}^{+\infty} B_{l} .
$$

As $B_{1} \subset B_{2} \subset B_{3} \subset \cdots$ and $(1)_{l}$ is satisfied by all $l$ we know that $N(U, U)$ is not thick, and consequently that $(X, S)$ is not weakly mixing.

We now check that $(X, S)$ satisfies property $(* *)$ in Lemma 5.3. As $x$ is recurrent, $(X, S)$ is transitive. For each neighborhood $V$ of $x$ there is $n \in \mathbb{N}$ such that $V_{n}=\left[C_{n}\right] \subset V$. Let $r \in \mathbb{Z}_{+}$and set $k=k_{n}+1$. By the definition of $F$, there exists infinitely many $l \in \mathbb{N}$ such that

$$
F(l+1)=(\phi(l+1), \varphi(l+1))=(n, r) .
$$

By the construction of $C_{l+1}$ (5.3) and (5.8), it is easy to see

$$
N\left(x, V_{n}\right) \supset\left\{a_{1}^{l}, a_{1}^{l}+a_{2}^{l}, \cdots, a_{1}^{l}+a_{2}^{l}+\cdots+a_{2(l+1)}^{l}\right\} .
$$

By (5.4), (5.2) and (5.8) we have

$$
a_{2 i}^{l}=q_{l, i}+k_{\phi(l+1)}=2^{k_{n}+1}\left(q_{l}+i\right)-\varphi(l+1)=2^{k}\left(q_{l}+i\right)-r .
$$


Thus,

$$
\begin{aligned}
N(V, V) & \supset N\left(V_{n}, V_{n}\right)=N\left(x, V_{n}\right)-N\left(x, V_{n}\right) \supset\left\{a_{2}^{l}, a_{4}^{l}, \cdots, a_{2(l+1)}^{l}\right\} \\
& =\left\{2^{k}\left(q_{l}+1\right)-r, 2^{k}\left(q_{l}+2\right)-r, \cdots, 2^{k}\left(q_{l}+l+1\right)-r\right\} .
\end{aligned}
$$

Hence $N_{k}(V, r)=\left\{n \in \mathbb{N}: 2^{k} n-r \in N(V, V)\right\} \supset\left\{q_{l}+1, q_{l}+2, \cdots, q_{l}+(l+1)\right\}$. This shows that $N_{k}(V, r)$ is thick. Hence $(X, S)$ is extremely scattering by Lemma 5.3 .

To finish the proof we must choose $p_{l, 1}, p_{l, 2}, \cdots, p_{l, l+1}, q_{l}$ such that $B_{l+1}$ satisfies $(1)_{l+1}$. There are many ways to do this, for example, we take

$q_{l}=k_{l}+\varphi(l+1)$ (by $(5.2)$ and (5.4) $a_{2 j}^{l}$ is determined by $\left.q_{l}\right)$,

$p_{l, 2}=\sum_{j=1}^{l+1} a_{2 j}^{l}+2 k_{l}+2$ and inductively we take

$p_{l, i} \geq \sum_{j=2}^{i-1} a_{2 j-1}^{l}+\sum_{j=1}^{l+1} a_{2 j}^{l}+2 k_{l}+2$ for $3 \leq i \leq l+1$. Finally, we take

$$
p_{l, 1} \geq \sum_{i=2}^{l+1} a_{2 i-1}^{l}+\sum_{j=1}^{l+1} a_{2 j}^{l}+2 k_{l}+2 .
$$

The reason we take $p_{l, 1}, p_{l, 2}, \cdots, p_{l, l+1}, q_{l}$ in the above form is that we want the following conditions to hold.

(i) if $1 \leq j \neq k \leq 2(l+1)$, then the four intervals

$$
\left[\left(a_{1}^{l}+\cdots+a_{j}^{l}\right) \pm B_{l}\right] \text { and }\left[\left(a_{1}^{l}+\cdots+a_{k}^{l}\right) \pm B_{l}\right]
$$

are disjoint with the gaps $\geq 2$ (see (5.6)) as $\max B_{l} \leq k_{l}$, where $\mathrm{A}$ ] is the convex hull of a finite set $A$ of $\mathbb{Z}_{+}$in $\mathbb{Z}_{+}$.

(ii) if $1 \leq i<j \leq 2(l+1)$ and $1 \leq k \leq 2(l+1)$, then the four intervals

$$
\left[\left(a_{1}^{l}+\cdots+a_{k}^{l}\right) \pm B_{l}\right] \text { and }\left[\left(a_{i+1}^{l}+\cdots+a_{j}^{l}\right) \pm B_{\phi(l+1)}\right]
$$

are disjoint with the gaps $\geq 2$ (see (5.6)) as $\max B_{\phi(l+1)}$, $\max B_{l} \leq k_{l}$.

(iii) if $1 \leq i_{1}<j_{1} \leq 2(l+1), 1 \leq i_{2}<j_{2} \leq 2(l+1)$ and $\left\{i_{1}, j_{1}\right\} \neq\left\{i_{2}, j_{2}\right\}$, then the four intervals

$$
\left[\left(a_{i_{1}+1}^{l}+\cdots+a_{j_{1}}^{l}\right) \pm B_{\phi(l+1)}\right] \text { and }\left[\left(a_{i_{2}+1}^{l}+\cdots+a_{j_{2}}^{l}\right) \pm B_{\phi(l+1)}\right]
$$

are disjoint with the gaps $\geq 2$ (see (5.6)) as $\max B_{\phi(l+1)} \leq k_{\phi(l+1)}$.

These in turn follow because (5.10), (5.11) and (5.12) below hold.

By choosing $p_{l, 1}, p_{l, 2}, \cdots, p_{l, l+1}, q_{l},(5.2)$ and (5.4) we know $a_{i}^{l} \geq 2 k_{l}+2, i=$ $1,2, \cdots, 2(l+1)$. Moreover, we have if $1 \leq j \neq k \leq 2(l+1)$, then

$$
\left|\left(a_{1}^{l}+\cdots+a_{j}^{l}\right)-\left(a_{1}^{l}+\cdots+a_{k}^{l}\right)\right| \geq 2 k_{l}+2 .
$$

By (5.4) and (5.9) we have $a_{1}^{l}-\left(a_{2}^{l}+a_{3}^{l} \cdots+a_{2(l+1)}^{l}\right) \geq 2 k_{l}+2$. Hence if $1 \leq i<j \leq 2(l+1)$ and $1 \leq k \leq 2(l+1)$, then

$$
\left|\left(a_{1}^{l}+\cdots+a_{k}^{l}\right)-\left(a_{i+1}^{l}+\cdots+a_{j}^{l}\right)\right| \geq 2 k_{l}+2 .
$$

Now we claim that if $1 \leq i_{1}<j_{1} \leq 2(l+1), 1 \leq i_{2}<j_{2} \leq 2(l+1)$ and $\left\{i_{1}, j_{1}\right\} \neq\left\{i_{2}, j_{2}\right\}$, then

$$
\left|\left(a_{i_{1}+1}^{l}+\cdots+a_{j_{1}}^{l}\right)-\left(a_{i_{2}+1}^{l}+\cdots+a_{j_{2}}^{l}\right)\right| \geq 2 k_{\phi(l+1)}+2 .
$$


Proof of the claim. Set $I_{1}=\left\{i_{1}+1, \ldots, j_{1}\right\}, I_{2}=\left\{i_{2}+1, \ldots, j_{2}\right\}$ and $I=\left(I_{1} \Delta I_{2}\right) \cap$ $\{3,5, \ldots, 2 l+1\}$.

Case 1: $I \neq \emptyset$.

Let $2 m+1=\max I$. Then

$$
\begin{aligned}
& \left|\left(a_{i_{1}+1}^{l}+\ldots+a_{j_{1}}^{l}\right)-\left(a_{i_{2}+1}^{l}+\ldots+a_{j_{2}}^{l}\right)\right| \\
& \geq a_{2 m+1}^{l}-\left(\sum_{i=1}^{l+1} a_{2 i}^{l}+\sum_{j=1}^{m-1} a_{2 j+1}^{l}\right) \quad(\text { by }(5.9) \text { and }(5.4)) \\
& \geq 2 k_{l}+2 \geq 2 k_{\phi(l+1)}+2 .
\end{aligned}
$$

Case 2: $I=\emptyset$.

If $I_{1} \cap\{3,5, \ldots, 2 l+1\}=\emptyset$, then $I_{1}=\left\{i_{1}\right\}, I_{2}=\left\{i_{2}\right\}$ and $i_{1} \neq i_{2}$ are even.

$$
\left|a_{i_{1}}^{l}-a_{j_{1}}^{l}\right|=\left|2^{k_{\phi(l+1)}+1} \frac{\left(i_{2}-i_{1}\right)}{2}\right| \geq 2 k_{\phi(l+1)}+2 .
$$

If $I_{1} \cap\{3,5, \cdots, 2 l+1\}=\{2 j+1,2 j+3, \cdots, 2 s+1\}$, then $I_{1}, I_{2} \in\{\{2 j$, $2 j+1, \cdots, 2 s+1\},\{2 j+1, \cdots, 2 s+1\},\{2 j, \cdots, 2 s+1,2(s+1)\},\{2 j+1, \cdots, 2 s+1$, $2(s+1)\}\}$. Then

$$
\begin{aligned}
& \left|\left(a_{i_{1}+1}^{l}+\ldots+a_{j_{1}}^{l}\right)-\left(a_{i_{2}+1}^{l}+\ldots+a_{j_{2}}^{l}\right)\right| \\
& \quad=a_{2 j}^{l} \text { or }\left|a_{2(s+1)}^{l}-a_{2 j}^{l}\right| \text { or } a_{2(s+1)}^{l} \text { or }\left|a_{2 j}^{l}+a_{2(s+1)}^{l}\right| \\
& \geq 2 k_{\phi(l+1)}+2 .
\end{aligned}
$$

This ends the proof of the claim.

Now we check that $B_{l+1}$ satisfies $(1)_{l+1}$.

Assume that there is $s$ such that $\{s, s-1\} \subset B_{l+1}$. By $(1)_{l}, s, s-1$ do not belong to $B_{l}$ simultaneously. As $\max B_{\phi(l+1)}, \max B_{l} \leq k_{l}$ and $a_{i}^{l} \geq 2 k_{l}+2(1 \leq$ $i \leq 2(l+1))$, for $1 \leq k \leq 2(l+1), 1 \leq i<j \leq 2(l+1)$

$\min \left\{\left(a_{1}^{l}+\cdots+a_{j}^{l}\right) \pm B_{l}\right\}, \min \left\{\left(a_{i+1}^{l}+\ldots+a_{j}^{l}\right) \pm B_{\phi(l+1)}\right\} \geq 2 k_{l}+2 \geq \max B_{l}+2$, we have (see (5.6))

$$
\begin{aligned}
\{s, s-1\} \subset & \bigcup_{k=1}^{2(l+1)}\left(\left(a_{1}^{l}+a_{2}^{l}+\cdots+a_{k}^{l}\right) \pm B_{l}\right) \\
& \cup \bigcup_{1 \leq i<j \leq 2(l+1)}\left(\left(a_{i+1}^{l}+a_{i+2}^{l}+\cdots+a_{j}^{l}\right) \pm B_{\phi(l+1)}\right) .
\end{aligned}
$$

By (5.10), (5.11), (5.12) and $B_{\phi(l+1)} \subset B_{l}$ there must exist $1 \leq i_{s} \leq j_{s} \leq 2(l+1)$ such that

$$
\{s, s-1\} \subset\left(a_{i_{s}}^{l}+a_{i_{s+1}}^{l}+\ldots+a_{j_{s}}^{l}\right) \pm B_{l} .
$$

From $(1)_{l}$ there is no $s$ with $\{s, s-1\} \subset B_{l}$. Thus

$$
s \in\left(a_{i_{s}}^{l}+\cdots+a_{j_{s}}^{l}\right)+B_{l} \text { and } s-1 \in\left(a_{i_{s}}^{l}+\cdots+a_{j_{s}}^{l}\right)-B_{l} .
$$

Since

$\min \left\{\left(a_{i_{s}}^{l}+\cdots+a_{j_{s}}^{l}\right)+B_{l}\right\}=\max \left\{\left(a_{i_{s}}^{l}+\cdots+a_{j_{s}}^{l}\right)-B_{l}\right\}=a_{i_{s}}^{l}+a_{i_{s+1}}^{l}+\cdots+a_{j_{s}}^{l}$, (5.13) is impossible as $1 \notin B_{l}$. That is, there is no $s$ with $\{s, s-1\} \subset B_{l+1}$. Hence $1 \notin B_{l+1}$ since $0 \in B_{l+1}$. 


\section{§. ApPENDiX}

In this Appendix we give the proofs of Proposition 3.5 and Example 3.7.

Proof of Proposition 3.5. Let $\pi:(X, T) \rightarrow\left(\Sigma_{K}, T_{K}\right)$ be an almost one-to-one extension, where $K=\left(k_{1}, k_{2}, \cdots\right)$. Note that for any $w=\left(w_{1}, w_{2}, \cdots\right) \in \Sigma_{K}$ and an open neighborhood $\left[w_{1}, w_{2}, \cdots, w_{l}\right]=\left\{t \in \Sigma_{K}: t_{i}=w_{i}, i=1,2, \cdots, l\right\}$ of $w$, one has

$$
N\left(w,\left[w_{1}, w_{2}, \cdots, w_{l}\right]\right)=\left(k_{1} \cdot k_{2} \cdots k_{l}\right) \mathbb{Z}_{+} .
$$

Hence each point of $\Sigma_{K}$ is a regular minimal point.

Now take $x \in X$ with $\pi^{-1} \pi(x)=\{x\}$. For any open neighborhood $U$ of $x$, there exists an open neighborhood $V$ of $\pi(x)$ such that $\pi^{-1} V \subset U$. Since $\pi(x)$ is a regular minimal point, we can find $k \in \mathbb{N}$ such that $N(\pi(x), V) \supset k \mathbb{Z}_{+}$. Therefore, $N(x, U) \supset N\left(x, \pi^{-1} V\right)=N(\pi(x), V) \supset k \mathbb{Z}_{+}$. This shows that $x$ is a regular minimal point. Moreover, each point in $I n j_{\pi}$ is a regular minimal point.

Conversely, let $x \in X$ be a regular minimal point. We have

Claim. For each open neighborhood $U$ of $x$, there exist a clopen (closed and open) neighborhood $A \subset U$ of $x$ and $l \geq 1$ such that $\left\{A, T A, \cdots, T^{l-1} A\right\}$ is a clopen partition of $X$ and $T^{l} A=A$.

Proof of the Claim. Take an open neighborhood $V$ of $x$ with $\operatorname{cl}(V) \subset U$. Since $x$ is a regular minimal point, there exists $k \in \mathbb{N}$ such that $N(x, V) \supset k \mathbb{Z}_{+}$. Thus $\omega\left(x, T^{k}\right) \subset \operatorname{cl}(V) \subset U$. Let $A=\omega\left(x, T^{k}\right)$. Then $x \in A \subset U$ and $\left(T^{i} A, T^{k}\right)$ is minimal, $0 \leq i \leq k-1$. If $T^{i} A \neq T^{j} A$, then $T^{i} A \cap T^{j} A=\emptyset$. As $T^{k} A=$ $\omega\left(T^{k} x, T^{k}\right)=\omega\left(x, T^{k}\right)=A$, there exists $0<l \leq k$ such that $A, T A, \cdots, T^{l-1} A$ are pairwise disjoint closed subsets of $X$ and $T^{l} A=A$. Since $\bigcup_{i=0}^{l-1} T^{i} A=\bigcup_{i=0}^{k-1} T^{i} A=$ $\omega(x, T)=X,\left\{A, T A, \cdots, T^{l-1} A\right\}$ is a clopen partition of $X$. This finishes the proof of the claim.

By the above claim, it is easy to construct inductively a sequence of clopen neighborhood $A_{i}$ of $x$ and $l_{i} \geq 1$ such that $A_{1} \supset A_{2} \supset \cdots, \bigcap_{i=1}^{\infty} A_{i}=\{x\}$ and $\left\{A_{i}, T A_{i}, \cdots, T^{l_{i}-1} A_{i}\right\}, i \in \mathbb{N}$, is a clopen partition of $X$. Since $A_{i} \subset A_{i+1}, l_{i} \mid l_{i+1}$ for each $i \in \mathbb{N}$.

Take $l_{0}=1$ and $k_{i}=\frac{l_{i}}{l_{i-1}}, i \in \mathbb{N}$. Let $K=\left(k_{1}, k_{2}, \cdots\right)$. Now we define $h: X \rightarrow \Sigma_{k}$ by

$$
h(x)_{i}=\left[\frac{j_{i}}{l_{i-1}}\right] \text {, where } 0 \leq j_{i} \leq l_{i}-1 \text { with } x \in T^{j_{i}} A_{i} \text {, for each } i \in \mathbb{N} .
$$

Now we show that $h:(X, T) \rightarrow\left(\Sigma_{K}, T_{K}\right)$ is an almost one-to-one extension.

First, we note that $x \in \bigcap_{i=1}^{\infty} T^{\sum_{j=1}^{i} l_{j-1} h(x)_{j}} A_{i}$. Hence

$$
T x \in \bigcap_{i=1}^{\infty} T^{\sum_{j=1}^{i} l_{j-1} h(x)_{j}+1} A_{i}=\bigcap_{i=1}^{\infty} T^{\sum_{j=1}^{i} l_{j-1}\left(T_{k} h(x)\right)_{j}} A_{i} .
$$

This shows that $h(T x)=T_{K} h(x)$. Second, for any $w=\left(w_{1}, w_{2}, \cdots\right) \in \Sigma_{K}$, since $T^{\sum_{j=1}^{i+1} l_{j-1} w_{j}} A_{i+1} \subset T^{\sum_{j=1}^{i} l_{j-1} w_{j}} A_{i}, i \in \mathbb{N}$, one has $\bigcap_{i=1}^{\infty} T^{\sum_{j=1}^{i} l_{j-1} w_{j}} A_{i} \neq \emptyset$. For any $z \in \bigcap_{i=1}^{\infty} T^{\sum_{j=1}^{i} l_{j-1} w_{j}} A_{i}, h(z)=w$. Hence $h$ is surjective. Third, since for each cylinder $\left[w_{1}, w_{2}, \cdots, w_{l}\right] \subset \Sigma_{K}, h^{-1}\left(\left[w_{1}, w_{2}, \cdots, w_{l}\right]\right)=\bigcap_{i=1}^{l} T^{\sum_{j=1}^{i} l_{j-1} w_{j}} A_{i}$ is an open set of $X, h$ is continuous. By the above discussion, we have seen that $h:(X, T) \rightarrow\left(\Sigma_{K}, T_{K}\right)$ is a factor map. 
Finally, since for each $j \in \mathbb{Z}_{+}, \bigcap_{i=1}^{+\infty} T^{j} A_{i}=\left\{T^{j} x\right\}$, i.e., $h^{-1}\left(h\left(T^{j} x\right)\right)=\left\{T^{j} x\right\}$ and $\operatorname{orb}(x, T)$ is dense in $X$, one knows that $I n j_{h}$ is dense in $X$. Hence $h$ is an almost one-to-one extension.

Now we present the construction. Note that for a word $A=(a(1), \ldots, a(n))$ and a sequence $y, A<y$ means that $A$ appears in $y$.

Proof of Example 3.7. We will construct a recurrent point $x \in\{0,1,2\}^{\mathbb{Z}_{+}}$such that $X=\operatorname{cl}(\operatorname{orb}(x, \sigma))$ is the system which we need. Let $y=(12212112 \cdots) \in\{1,2\}^{\mathbb{Z}_{+}}$ be the Morse sequence. It is known [GH1] that Morse sequence $y$ has the following property: $w^{3} \nless y$ for any finite word $w$.

Define $F: \mathbb{N} \longrightarrow \mathbb{N} \times \mathbb{Z}_{+}$by $F(s)=(\phi(s), \varphi(s))$ such that $\phi(s) \leq s$, and for any $(k, r) \in \mathbb{N} \times \mathbb{Z}_{+}$there exist infinitely many $j \in \mathbb{N}$ with $F(j)=(k, r)$.

We use induction to construct a recurrent point $x=\left(x_{0}, x_{1}, \cdots\right) \in\{0,1,2\}^{\mathbb{Z}_{+}}$. To do this, we construct inductively a sequence of finite words $A_{i}$ such that $A_{i+1}$ begins with $A_{i}$ and $x$ is the limit of $A_{i}$. To begin with we let $A_{1}=0$ and $n_{1}=$ $\left|A_{1}\right|=1$. Assume that we have constructed $A_{l}$ for $1 \leq l \leq k$. Set $n_{l}=\left|A_{l}\right|$. For $l=k+1$, put

$$
\begin{aligned}
A_{k+1}= & A_{k} y\left[0 ; m_{k}-1\right] A_{\phi(k)} y\left[m_{k} ; m_{k}+9 n_{\phi(k)}-1\right] \cdots \\
& A_{\phi(k)} y\left[m_{k}+9 i n_{\phi(k)} ; m_{k}+9(i+1) n_{\phi(k)}-1\right] \cdots \\
& A_{\phi(k)} y\left[m_{k}+9(k-1) n_{\phi(k)} ; m_{k}+9 k n_{\phi(k)}-1\right] A_{\phi(k)},
\end{aligned}
$$

where $m_{k}=10 t_{k} n_{\phi(k)}-n_{k}+\varphi(k)$ and $t_{k}$ is large enough with $m_{k} \geq \frac{8}{9} n_{k+1}$ (note that $\left.n_{k+1}=n_{k}+m_{k}+(10 k+1) n_{\phi(k)}\right)$.

Let $x=\lim _{k \rightarrow+\infty} A_{k}$ and $X=\operatorname{cl}(\operatorname{orb}(x, \sigma))$. By the construction of $A_{k}$, it is easy to see that for any given $(k, r) \in \mathbb{N} \times \mathbb{Z}_{+}$and $s$ with $F(s)=(k, r)$,

$A_{s+1}=A_{s} y\left[0 ; m_{s}-1\right] A_{k} y\left[m_{s} ; m_{s}+9 n_{k}-1\right] A_{k} y\left[m_{s}+9 n_{k}, m_{s}+18 n_{k}-1\right] \ldots A_{k}$.

As $n_{s}+m_{s}+10 i n_{k}=n_{s}+10 t_{s} n_{k}-n_{s}+r+10 i n_{k}=10 n_{k}\left(t_{s}+i\right)+r$ and $N_{k}(x, U, r)=\left\{n \in \mathbb{Z}_{+}: T^{k n+r}(x) \in U\right\}$, we have

$$
N_{10 n_{k}}\left(x,\left[A_{k}\right], r\right) \supset \bigcup_{s \text { with } F(s)=(k, r)}\left\{t_{s}, t_{s}+1, \cdots, t_{s}+s-1\right\} .
$$

Therefore, $(X, \sigma) \in \mathcal{M}^{\perp}$ by Theorem 3.4.

Now, we show that $(X, \sigma)$ has no periodic point. First, we have

Claim 1: For every $k \in \mathbb{N}$, one has

$(1)_{k}$ There is no finite word $w$ such that $t=|w| \leq \frac{n_{k}}{3}$ and $w^{3}=A_{k}[0 ; 3 t-1]$.

$(2)_{k}$ There is no finite word $w$ such that $t=|w| \leq \frac{n_{k}}{3}$ and $w^{3}=A_{k}\left[n_{k}-3 t\right.$; $\left.n_{k}-1\right]$.

Proof of Claim 1. (1) 1 is obvious. Assume that $(1)_{k}$ holds for $1 \leq k \leq l$ and (1) $k$ fails for $k=l+1$. Then there exists a finite word $w$ such that $t=|w| \leq \frac{n_{k}}{3}$ and $w^{3}=A_{k}[0 ; 3 t-1]$. By $(1)_{l}, n_{l+1} \geq 3 t>n_{l}$.

Since $m_{l} \geq \frac{8}{9} n_{l+1}$, one has $2 t<n_{l}+m_{l}$. As $w(0)=A_{k}(0)=0,0 \nless y$ and $A_{k}(2 t)=w(0)=0$, one has $2 t<n_{l}$. Moreover, $l \geq 2$ since $t \geq 1$ and $n_{1}=1$.

Since $m_{l-1}+n_{l-1}>\frac{n_{l}}{2}>t>\frac{n_{l}}{3}>n_{l-1}$ and $A_{l}\left[n_{l-1} ; n_{l-1}+m_{l-1}-1\right]=$ $y\left[0 ; m_{l-1}-1\right]$, one has $0=w(0)=A_{l}(t)<y$, a contraction, as $0 \nless y$. 
$(2)_{1}$ is obvious. Assume that $(2)_{k}$ holds for $1 \leq k \leq l$ and fails for $k=l+1$. Then there exists a finite word $w$ such that $t=|w| \leq \frac{n_{k}}{3}$ and $w^{3}=A_{k}\left[n_{k}-3 t ; n_{k}-1\right]$. By $(2)_{\phi(l)}, n_{l+1} \geq 3 t>n_{\phi(l)}$. There are two cases for $t$.

Case 1: $t<n_{\phi(l)}$. Since $A_{k}\left(n_{k}-2 t-1\right)=w(t-1)=A_{k}\left(n_{k}-1\right)=0$ and $0 \nless y$, one has $2 t<n_{\phi(l)}$. This shows that $\frac{n_{\phi(l)}}{2}>t>\frac{n_{\phi(l)}}{3}$. Moreover, one has $\phi(l) \geq 2$ and

$$
n_{\phi(l)-1} \leq \frac{n_{\phi(l)}}{2}-1<n_{\phi(l)}-t-1<\frac{2 n_{\phi(l)}}{3}-1 \leq n_{\phi(l)-1}+m_{\phi(l)-1} .
$$

Thus $A_{n_{\phi(l)}}\left(n_{\phi(l)}-t-1\right)<y\left[0 ; m_{\phi(l)-1}-1\right]$, a contradiction, as $A_{n_{\phi(l)}}\left(n_{\phi(l)}-t-1\right)=$ $w(t-1)=0$ and $0 \nless y$.

Case 2: $t \geq n_{\phi(l)}$. Since $w\left[t-n_{\phi(l)} ; t-1\right]=A_{\phi(l)}, A_{\phi(l)}(0)=A_{\phi(l)}\left(n_{\phi(l)}-1\right)=0$ and $0 \nless y$, there exists $i \in \mathbb{Z}_{+}$such that

$$
\begin{aligned}
w= & y\left[m_{l}+9 i n_{\phi(l)} ; m_{l}+9(i+1) n_{\phi(l)}-1\right] A_{\phi(l)} \cdots \\
& A_{\phi(l)} y\left[m_{l}+9(l-1) n_{\phi(l)} ; m_{l}+9 l n_{\phi(l)}-1\right] A_{\phi(l)} .
\end{aligned}
$$

It follows by the above equality that $n_{k}-t \geq n_{l}+m_{l}$. Hence $t \leq n_{k}-\left(n_{l}+m_{l}\right) \leq$ $\frac{1}{9} n_{k} \leq \frac{1}{8} m_{l}$. Moreover, $n_{k}-3 t>n_{l}$. Noting that $A_{k}\left[n_{k}-3 t ; n_{k}-1\right]=w^{3}$ and $w\left[9 n_{\phi(l)} ; 10 n_{\phi(l)}-1\right]=A_{\phi(l)}$, one has $A_{k}\left[n_{k}-3 t+9 n_{\phi(l)} ; n_{k}-3 t+10 n_{\phi(l)}-1\right]=A_{\phi(l)}$.

As $A_{\phi(l)}(0)=A_{\phi(l)}\left(n_{\phi(l)}-1\right)=0$ and $0 \nless y$, one gets $n_{k}-3 t+9 n_{\phi(l)} \geq n_{l}+m_{l}$. Let $u=y\left[m_{l}+9 i n_{\phi(l)} ; m_{l}+9 \ln n_{\phi(l)}-1\right]$. If $n_{k}-3 t \geq n_{l}+m_{l}$, then

$$
\begin{aligned}
w^{3}= & A_{k}\left[n_{k}-3 t ; n_{k}-1\right] \\
= & y\left[m_{l}+9 j n_{\phi(l)} ; m_{l}+9(j+1) n_{\phi(l)}-1\right] A_{\phi(l)} \cdots \\
& A_{\phi(l)} y\left[m_{l}+9(l-1) n_{\phi(l)} ; m_{l}+9 l n_{\phi(l)}-1\right] A_{\phi(l)},
\end{aligned}
$$

where $j=l-3(l-i+1)+1$. Hence $u^{3}=y\left[m_{l}+9 j n_{\phi(l)} ; m_{l}+9 l n_{\phi(l)}-1\right]<y$, a contradiction.

If $n_{k}-3 t<n_{l}+m_{l}$, then

$$
\begin{aligned}
w^{3}= & A_{k}\left[n_{k}-3 t ; n_{k}-1\right] \\
= & y\left[m_{l}-9 n_{\phi(l)} ; m_{l}-1\right] A_{\phi(l)} y\left[m_{l} ; m_{l}+9 n_{\phi(l)}-1\right] A_{\phi(l)} \cdots \\
& A_{\phi(l)} y\left[m_{l}+9(l-1) n_{\phi(l)} ; m_{l}+9 \ln n_{\phi(l)}-1\right] A_{\phi(l)} .
\end{aligned}
$$

Hence $u^{3}=y\left[m_{l}-9 n_{\phi(l)} ; m_{l}+9 \ln _{\phi(l)}-1\right]<y$, a contradiction. This ends the proof of claim 1 .

Second, we have

Claim 2: There is no finite word $w$ with $w^{8}<x$.

Proof of Claim 2. Assume that there exists a finite word $w$ with $w^{8}<x$. Let $k$ be the least natural number with $w^{8}<A_{k}$. Clearly, $k \geq 2$. Set $|w|=t$. Then there is $0 \leq l \leq n_{k}-8 t$ with $A_{k}[l ; l+8 t-1]=w^{8}$. By the choosing of $k$, one knows $w^{8} \nless A_{k-1}$. Therefore, $l+8 t>n_{k-1}$.

Let $w=w(0) w(1) \cdots w(t-1)$ and define

$$
P^{j} w=w(j) w(j+1) \cdots w(t-1) w(0) \cdots w(j-1), \quad j=1,2, \cdots, t-1 .
$$


Since 0 appears in both endpoint of $A_{m}$ for each $m \in \mathbb{N}, 0 \nless y$ and $w^{3} \nless y$, one has $w, P w, P^{2} w, \cdots, P^{t-1} w \nless y$. There are two cases for $l$.

Case 1: $l<n_{k-1}$. Since $m_{k-1} \geq \frac{8}{9} n_{k}, l+6 t<n_{k-1}+m_{k-1}$. By $(1)_{k-1}$ of claim $1, l+3 t \geq n_{k-1}$. This shows that $n_{k-1} \leq l+3 t<l+6 t<n_{k-1}+m_{k-1}$. Therefore, $w^{3}<A_{k}\left[n_{k-1} ; n_{k-1}+m_{k-1}-1\right]=y\left[0 ; m_{k-1}-1\right]$, a contradiction.

Case 2: $l \geq n_{k-1}$. Since $w \nless y, l \geq n_{k-1}+m_{k-1}-t$. If $t<n_{\phi(k-1)}$. By Claim 1 and $w, P w, P^{2} w, \cdots, P^{t-1} w \nless y$, one has $w^{8}<A_{\phi(k-1)}$, a contradiction since $\phi(k-1)<k$. Thus $t \geq n_{\phi(k-1)}$. Since $w \nless y$, there exists $0 \leq m \leq k-1$ such that $l<s_{k} \leq l+2 t$, where $s_{k}=n_{k-1}+m_{k-1}+10 m n_{\phi(k-1)}$. Set $v=A_{k}\left[s_{k} ; s_{k}+t-1\right]$. Then $v^{6}=A_{k}\left[s_{k} ; s_{k}+6 t-1\right]$.

Since $v\left[0 ; n_{\phi(k-1)}-1\right]=A_{\phi(k-1)}, A_{\phi(k-1)}(0)=A_{\phi(k-1)}\left(n_{\phi(k-1)}-1\right)=0$ and $0 \nless y$, we can find $n \in \mathbb{Z}_{+}$such that

$$
\begin{aligned}
v= & A_{\phi(k-1)} y\left[m_{k-1}+9 m n_{\phi(k-1)} ; m_{k-1}+9(m+1) n_{\phi(k-1)}-1\right] \cdots \\
& A_{\phi(k-1)} y\left[m_{k-1}+9(m+n) n_{\phi(k-1)} ; m_{l}+9(m+n+1) n_{\phi(k-1)}-1\right] .
\end{aligned}
$$

Put $u=y\left[m_{k-1}+9 m n_{\phi(k-1)} ; m_{k-1}+9(m+n+1) n_{\phi(k-1)}-1\right]$. Since $v^{6}=$ $A_{k}\left[s_{k} ; s_{k}+6 t-1\right]$, one has $u^{6}<y$ which contradicts $u^{3} \nless y$. This finishes the proof of Claim 2.

Thus, $(X, \sigma)$ has no periodic point by Claim 2 .

\section{ACKNOWLEDGEMENTS}

We thank the referee of the paper for his very careful reading and many valuable suggestions.

\section{REFERENCES}

[A] J. Auslander, Minimal Flows and Their Extensions, North-Holland Mathematics Studies, 153. North-Holland Publishing Co., Amsterdam, 1988. MR 89m:54050

[AG] E. Akin and E. Glasner, Residual properties and almost equicontinuity, J. d'Anal. Math., 84 (2001), 243-286. MR 2002f:37020

[B] J. Banks, Regular periodic decomposition for topologically transitive maps, Ergod. Th. and Dynam. Sys., 17 (1997), 505-529. MR 98d:54074

[BHM] F. Blanchard, B. Host and A. Maass, Topological complexity, Ergod. Th. and Dynam. Sys., 20 (2000), 641-662. MR 2002b:37019

[DY] T. Downarowicz and X. Ye, When every point is either transitive or periodic, Colloq. Math., 93 (2002), 137-150. MR 2003g:37015

[F] H. Furstenberg, Disjointness in ergodic theory, minimal sets, and a problem in Diophantine approximation, Math. Systems Theory, 1 (1967), 1-49. MR 35:4369]

[GH1] W. Gottschalk and G. Hedlund, A characterization of the Morse minimal set, Proc. Amer. Math. Soc., 15 (1964), 70-74. MR 28:1609

[GH2] W. Gottschalk and G. Hedlund, Topological Dynamics, Amer. Math. Soc. Colloq., Vol. XXXVI, 1955. MR 17:650e

[HY] W. Huang and X. Ye, An explicit scattering, nonweakly mixing example and weak disjointness, Nonlinearity, 15 (2002), 849-862. MR 2003b:37016

[K] J. Kelly, General Topology, Graduate Texts in Mathematics, 27, 1955.

$[\mathrm{M}]$ D. McMahon, Relativized weak disjointness and relatively invariant measures, Trans. Amer. Math. Soc., 236 (1978), 225-237. MR 57:7557

[P] K. Petersen, Disjointness and weak mixing of minimal sets, Proc. Amer. Math. Soc., 24 (1970), 278-280. MR 40:3522

[SY] S. Shao and X. Ye, $\mathcal{F}$-mixing and weak disjointness, Topology and its Application, 135 (2004), 231-247. 
[Wa] P. Walters, An introduction to ergodic theory, Graduate Texts in Mathematics, 79, Springer-Verlag, New York, Berlin, 1982. MR 84e:28017

[We] B. Weiss, A survey of generic dynamics, LMS Lecture Note Series, 277, Cambridge University Press, 2000, 273-291. MR 2001j:37008

Department of Mathematics, University of Science and Technology of China, Hefei, Anhui, 230026, People's Republic of China

E-mail address: wenh@mail.ustc.edu.cn

Department of Mathematics, University of Science and Technology of China, Hefei, Anhui, 230026, People's Republic of China

E-mail address: yexd@ustc.edu.cn 